\title{
Metabolic cobordisms and the simplest perturbative Chern-Simons 3-manifold invariant
}

\author{
Clifford Henry Taubes
}

1 Introduction. The perturbative, Chern-Simons three-manifold invariants were predicted by Witten [8] and then defined by Axelrod and Singer ([1] and [2]). Kontsevich [4] has given a second realization of Witten's predictions; and the simplest of Kontsevich's invariants is the subject of this article, and its prequel, [7].

As described in the first article, [7], the simplest of Kontsevich's perturbative Chern-Simons invariants is defined for compact, oriented 3-manifolds $\mathrm{M}$ which have the rational homology of $S^{3}$. The invariant, $I_{2}(M)$, is computed by an integral

$$
I_{2}(M)=\int_{M \times M} \omega \wedge \omega \wedge \omega
$$

where $\omega$ is a closed 2 -form on $M \times M$ with a prescribed singularity on

$$
\Sigma_{M} \equiv \Delta_{M} \cup\left(p_{0} \times M\right) \cup\left(M \times p_{0}\right) .
$$

Here, $\Delta_{M} \subset M \times M$ is the diagonal, and $p_{0} \in M$ is a chosen base point.

The reader is referred to Section 2 of [7] and Definition 2.8 of [7] for the details. (This article is a sequel to [7].) Suffice it to say that $H_{\text {DeRham }}^{2}\left(M \times M-\Sigma_{M}\right) \approx \mathbb{R}$, and $\omega$ is a generator of this group. In particular, $\omega$ restricts to every linking 2 -sphere around $\Sigma_{M}$ as 2-form with total volume 1. None-the-less, $\omega$ is constrained so that $\omega \wedge \omega=0$ near $\Sigma_{M}$, thus insuring that the integral in (1.1) is well defined.

\section{a) Cobordisms and $I_{2}$.}

Let $M_{0}$ and $M_{1}$ be a pair of compact, oriented, 3-manifolds with the rational homology of $S^{3}$. An oriented cobordism, $W$, between $M_{0}$ and $M_{1}$ is a compact, oriented 4-manifold with boundary; and that boundary should be the disjoint union of $M_{0}$ and $M_{1}$. Furthermore, the induced boundary orientation (using the outward pointing normal) should be correct for $M_{1}$ and wrong for $M_{0}$. If $W$ is also a spin manifold, then the cobordism is called a spin cobordism.

Theorem 2.9 in [7] gives a set of conditions on the spin cobordism $W$ which imply $I_{2}\left(M_{0}\right)=I_{2}\left(M_{1}\right)$. In particular, one condition in [7]'s Theorem 2.9 required that $W$ have the rational homology of $S^{3}$. It is the purpose of this

Supported in part by the National Science Foundation. Dedicated to Raoul Bott on the occasion of his 70 th birthday. 
article to greatly relax this condition. The relaxed conditions are stated below in Theorem 1.2. Here is a corollary of Theorem 1.2:

THEOREM 1.1. The invariant $I_{2}(\cdot)$ equals zero on a 3-manifold with the integral homology of $S^{3}$.

The full statement of Theorem 1.2 requires the following digression to introduce some necessary terminology. To start the digression, introduce tor $\left(\mathrm{H}_{2}\right) \subset$ $H_{2}(W ; \mathbb{Z})$ to denote the torsion sub-group. Next, recall that there is a natural, symmetric, bilinear form on $\underline{H}_{2}(W ; \mathbb{Z}) \equiv H_{2}(W ; \mathbb{Z}) / \operatorname{tor}\left(H_{2}\right)$, this being the intersection pairing. This form is non-degenerate, but not in general unimodular. (It is unimodular if $M_{0}$ and $M_{1}$ have the integral homology of $S^{3}$.) The intersection form, $\beta$, on $\underline{H}_{2}(W ; \mathbb{Z})$ will be called equivalent to a sum of metabolics if it is conjugate under $G L(\cdot, \mathbb{Z})$ to

$$
\oplus_{a} H\left(m_{a}\right)
$$

where $H(m)$ for $m \in \mathbb{Z}$ is the symmetric, $2 \times 2$ matrix with zero on the diagonal and with $m$ in the off diagonal entries. For example, the compliment of a pair of disjoint, open balls in $S^{2} \times S^{2}$ is a spin cobordism between $S^{3}$ and $S^{3}$ whose intersection form is conjugate to either $H(1)$ or to $H(-1)$, depending on the orientation.

End the digression.

THEOREM 1.2. Let $M_{0}$ and $M_{1}$ be compact, oriented, 3-manifolds with the rational homology of $S^{3}$. Let $W$ be an oriented, spin cobordism between $M_{0}$ and $M_{1}$. Suppose that:

1) The intersection form of $W$ is equivalent to a sum of metabolics.

2) The inclusions of both $M_{0}$ and $M_{1}$ into $W$ induce injective maps on $H_{1}(\cdot ; \mathbb{Z} / 2)$.

Then $I_{2}\left(M_{0}\right)=I_{2}\left(M_{1}\right)$.

Theorem 1.2 is an immediate corollary to Theorem 1.3, below. The statement of Theorem 1.3 requires the following 2-part digression. For Part 1 of the digression, consider a compact, oriented 3-manifold $M$ with the rational homology of $S^{3}$. Fix a point $p_{0} \in M$. Then, introduce from Definition 2.3 in [7] the notion of a singular frame for $T^{*} M$. (This is a frame for $T^{*}\left(M-p_{0}\right)$ with a prescribed singularity at $p_{0}$.) As in Lemma 2.4 of [7], let $c$ denote the set of homotopy classes of singular frames for $T^{*} M$. Define an equivalence relation on $c$ by declaring $\zeta$ and $\zeta^{\prime}$ to be equivalent when $\zeta=g \cdot \zeta^{\prime}$ where $g$ is a degree zero map from $M$ to $S O(3)$. Let $\underline{c}$ denote the resulting set of equivalence classes. Finally, use Definition 2.8 of [7] to identify a canonical element $c_{M} \in \underline{c}$.

For Part 2 of the digression, let $W$ be a compact, oriented 4-manifold with boundary and suppose that $M$, as above, is a component of $\partial W$. Let $K(M ; W)$ denote the cokernel of the restriction homomorphism $H^{1}(W ; \mathbb{Z} / 2) \longrightarrow$ $H^{1}(M ; \mathbb{Z} / 2)$. As in $(2.12)$ of [7], introduce the homomorphism $l_{W}: c \longrightarrow K(M ; W)$.

End the digression. 
THEOREM 1.3. Let $M_{0}$ and $M_{1}$ be compact, oriented, 3-manifolds with the rational homology of $S^{3}$. Let $W$ be an oriented, spin cobordism between $M_{0}$ and $M_{1}$. Suppose that:

1) The intersection form of $W$ is equivalent to a sum of metabolics.

2) Both $c_{M_{0}}$ and $c_{M_{1}}$ are represented by $c$ with $l_{W}(c)=0$.

Then $I_{2}\left(M_{0}\right)=I_{2}\left(M_{1}\right)$.

\section{b) Proof of Theorem 1.1.}

Orient $S^{3}$ as the boundary of the unit ball in $\mathbb{C}^{2}$. Let $P$ denote the Poincaré homology sphere. This is a quotient of $S^{3}$ by a certain finite subgroup of $S O(4)$, and as such, inherits an orientation from $S^{3}$. (Note that $P$ is an integral homology sphere.)

Note that $P$ is spin cobordant to $S^{3}$, and there is such a spin cobordism with signature divisable by 8 (See, e.g., [3]). (The signature of a 4-manifold is the number of positive eigenvalues minus the number of negative eigenvalues in the intersection form after diagonalizing the latter over $\mathbb{Q}$.)

Let $M$ be a compact, oriented 4-manifold with the integral homology of $S^{3}$. There is an oriented, spin cobordism between $M$ and $S^{3}$, and the index of such a cobordism is automatically divisible by 8 (again, see [3].) Thus, there is a spin cobordism between $M$ and $S^{3}$ or between $M$ and $P$ with signature divisable by 16. Let $W$ denote the afore-mentioned cobordism. If the signature of $W$ is not zero, then the connect sum of $W$ with some number of $K 3$ surfaces (with the appropriate orientations) gives a new spin cobordism between $M$ and $S^{3}$ or $P$ which has signature zero. This will now be assumed.

The intersection form of $\mathrm{W}$ is a signature zero, unimodular, even, symmetric matrix. (The form is even if $\beta(x, x) \in 2 \mathbb{Z}$ for all $x$.) Such a form is equivalent over $G l(\cdot, \mathbb{Z})$ to a sum of metabolics as in (1.3) with all $m_{a}= \pm 1$ (once again, see [3]). Thus, the first condition of Theorem 1.2 is met with $M_{0}=M$ and with $M_{1}=S^{3}$ or $P$. The second condition of Theorem 1.2 is also met because both $M_{0}$ and $M_{1}$ have trivial homology. It follows that $I_{2}(M)=I_{2}\left(S^{3}\right)$ or else $I_{2}(M)=I_{2}(P)$. Now $I_{2}\left(S^{3}\right)=0$ (Theorem 2.9 in [7]) so Theorem 1.1 will follow with a demonstration that $I_{2}(P)=0$.

With the preceding understood, let $\underline{P}$ denote the Poincare homology sphere with the opposite orientation. Then $I_{2}(\underline{P})=I_{2}(P)$ since $P \cup P$ bounds a spin cobordism with index divisable by 16 . However, unless $I_{2}(P)=0$, this conclusion is incompatible with

LEMMA 1.4. Let $M$ be a compact, oriented 3-manifold with the integral homology of $S^{3}$. Let $\underline{M}$ denote the manifold $M$ with reversed orientation. Then $I_{2}(M)=-I_{2}(\underline{M})$.

Proof. The form $\omega$ for the computation of $I_{2}(M)$ is constructed after choosing a singular frame, $\zeta$, for $T^{*} M$ in the class of $c_{M}$. (See Proposition 2.5 in [7].) The frame $\zeta \equiv\left(\zeta_{1}, \zeta_{2}, \zeta_{3}\right)$ must be compatible with the orientation of $M$. With this point understood, then $\zeta^{\prime} \equiv\left(-\zeta_{1}, \zeta_{2}, \zeta_{3}\right)$ will be an oriented, singular frame for $T^{*} \underline{M}$, which is in the class $c_{\underline{M}}$ (see Proposition 2.7 and Definition 2.8 of 
[7].) The form $-\omega$ will result from the constructions in [7] using the frame $\zeta^{\prime}$. Meanwhile, the orientation of $M \times M$ is not sensitive to the choice of orientation for $M$.

\section{c) An outline for proving Theorem 1.3.}

Here is a 4 step program for proving Theorem 1.3:

Step 0: $\quad$ Start with a cobordism between $M_{0}$ and $M_{1}$ which obeys the conditions of Theorem 1.3. Appeal to Proposition 3.2 in [7] to find a cobordism $W$ between $M_{0}$ and $M_{1}$ which decomposes as $W_{1} \cup W_{2} \cup W_{3}$ with the following properties:

1) $\partial W_{1}=M_{0} \cup M_{0}^{\prime}, \partial W_{2}=M_{0}^{\prime} \cup M_{1}^{\prime}$, and $\partial W_{3}=M_{1}^{\prime} \cup M_{1}$, where $M_{0}^{\prime}$ and $M_{1}^{\prime}$ are compact, oriented 3-manifolds with the rational homology of $S^{3}$.

2) $W_{1,2,3}$ are oriented, spin manifolds.

3) Both $W_{1}$ and $W_{3}$ have the rational homology of $S^{3}$. Meanwhile $W_{2}$ has vanishing first and third Betti numbers; and $W_{2}$ has a proper Morse function with no index 1 or index 3 critical points.

4) Both $c_{M_{0}}$ and $c_{M_{1}}$ are represented by elements in the kernel of $\iota_{W}$.

Step 1: Construct a compact, oriented 7-manifold with boundary $Z \subset$ $W \times W$. The boundary of this $Z$ is the disjoint union of $M_{0} \times M_{0}, M_{1} \times M_{1}$ and some number of copies of $S^{3} \times S^{3}$. Note that

$$
Z \equiv Z_{1} \cup Z_{2} \cup Z_{3}
$$

where $Z_{i} \subset W_{i} \times W_{i}$ are compact submanifolds with boundary. The $Z_{i}$ are described in Section $3 \mathrm{~g}$ of [7]. (The boundary of $Z_{1}, \partial Z_{1}$, is the disjoint union of $M_{0} \times M_{0}$ and $M_{0}^{\prime} \times M_{0}^{\prime}$ plus copies of $S^{3} \times S^{3}$. Meanwhile $\partial Z_{2}$ contains $M_{0}^{\prime} \times M_{0}^{\prime}, M_{1}^{\prime} \times M_{1}^{\prime}$ and copies of $S^{3} \times S^{3}$. Finally, $\partial Z_{3}$ is the disjoint union of $M_{1}^{\prime} \times M_{1}^{\prime}, M_{1} \times M_{1}$ plus copies of $S^{3} \times S^{3}$. In addition, $Z_{1}$ and $Z_{2}$ are glued together along $M_{0}^{\prime} \times M_{0}^{\prime}$, while $Z_{2}$ and $Z_{3}$ are glued together across $M_{1}^{\prime} \times M_{1}^{\prime}$.)

Step 2: Inside $Z$, find an oriented, dimension 4 subvariety $\Sigma_{Z}$ with $\partial Z$ being the disjoint union of $\Sigma_{M_{0}}, \Sigma_{M_{1}}$ and copies of $\Sigma_{S^{3}}$, one in each $S^{3} \times S^{3}$ boundary component. Note that

$$
\Sigma_{Z}=\Sigma_{Z_{1}} \cup \Sigma_{Z_{2}} \cup \Sigma_{Z_{3}}
$$

where $\Sigma_{Z_{1,3}}$ are described in [7] (see Eq. (4.10) in [7] and consider Sections 4d, 4e and 10 in [7].) The subvariety $\Sigma_{Z_{2}} \subset Z_{2}$ is constructed here, and the details of the construction of $\Sigma_{Z_{2}}$ account for most of the length of this article. 
Step 3: Find a closed 2-form, $\omega_{Z}$, on $Z-\Sigma_{Z}$ with the following properties:

1) The 2-form $\omega_{Z}$ should restrict to $M_{0} \times M_{0}-\Sigma_{M_{0}}$ as the 2-form used in (1.1) for computing $I_{2}\left(M_{0}\right)$. Its restriction to $M_{1} \times M_{1}-\Sigma_{M_{1}}$ should give the 2-form used in (1.1) for computing $I_{2}\left(M_{1}\right)$.

2) The 2-form $\omega_{Z}$ should restrict to each $S^{3} \times S^{3}-\Sigma_{S^{3}}$ as the 2-form used for (1.1) for computing $I_{2}\left(S^{3}\right)=0$.

3) The triple wedge product $\omega_{Z} \wedge \omega_{Z} \wedge \omega_{Z}$ should vanish near $\Sigma_{Z}$.

Step 4: Given that $\omega_{Z}$ exists as prescribed above, use Stokes' theorem as in (2.28) of [7] to prove that $I_{2}\left(M_{0}\right)=I_{2}\left(M_{1}\right)$.

\section{d) Issues in the construction of $Z, \Sigma_{Z}$ and $\omega_{Z}$.}

Compare the outline above with the outline in Section $2 k$ of [7] for the proof of [7]'s Theorem 2.9 and note the similarity between the two strategies. The construction of $Z_{2} \subset W_{2} \times W_{2}$ mimics the construction of $Z_{1}$ and $Z_{3}$ in [7]. In all cases $Z$. is given as $F^{-1}(0)$ for a function $F \equiv\left(\pi_{R}^{*} f-\pi_{L}^{*} f\right)$ on $W . \times W$. which is constructed from an appropriately chosen function $f: W . \longrightarrow[0,1]$. Here $\pi_{R, L}: W . \times W . \longrightarrow W$. are the left and right projections. (As in [7], the extra $S^{3} \times S^{3}$ boundary components of $Z$. are in 1-1 correspondence with the critical points of the function $f$.)

The construction of $\Sigma_{Z_{2}}$ here also mimics the construction of $\Sigma_{Z_{1}}$ and $\Sigma_{Z_{3}}$ in [7] in that all are given as $\Sigma_{Z}=\Delta_{Z} \cdot \cup E_{L} \cup E_{R} \cup E_{-} \cup E_{+}$. Here, as in [7], $\Delta_{Z} \cdot \subset W . \times W$. is the diagonal, $E_{L, R}$ are sections for the projections $\pi_{L, R}$, and $E_{ \pm}$are certain subvarieties which are constructed with the help of a pseudogradient vector field for the function $f$. As in [7], the variety $\Sigma_{Z}$ will restrict to each component $M \times M \subset \partial Z$ as $\Sigma_{M}$.

Furthermore, the variety $\Sigma_{Z_{2}}$ is constructed so that the conclusions of Lemma 4.1 hold for $\Sigma_{Z}$. This lemma gives necessary and sufficient conditions for $Z_{2}-\Sigma_{Z_{2}}$ to admit a closed 2-form, $\omega_{Z_{2}}$, whose restriction to each component $M \times M \subset \partial Z_{2}$ is a generator of $H^{2}\left(M \times M-\Sigma_{M}\right)$. The analogous closed forms, $\omega_{Z_{1}}$ and $\omega_{Z_{3}}$ are constructed on $Z_{1}-\Sigma_{Z_{1}}$ and $Z_{3}-\Sigma_{Z_{3}}$ in Section 10 of [7]. The form $\omega_{Z}$ in (1.7) is defined so that its restriction to $Z$. $=Z_{1}, Z_{2}$, and $Z_{3}$ is equal to the corresponding $\omega_{Z}$.

The construction of a 2 -form $\omega_{Z_{2}}$ as above which satisfies the first two requirements of (1.7) follows essentially the same plan as used in Section 10 of [7] to construct $\omega_{Z_{1}}$ and $\omega_{Z_{3}}$. The most difficult requirement to satisfy is the third requirement of (1.7). The new difficulty, not present in [7], is the fact that the restriction map $\iota^{*}: H^{2}\left(Z_{2}\right) \longrightarrow H^{2}\left(\Sigma_{Z_{2}}\right)$ is not surjective when $W$ has rational $H^{2}$.

Indeed, the arguments in [7] can be followed with minor modifications to construct a 2-form, $\underline{\omega}_{Z_{2}}$, on the compliment of $\Sigma_{Z_{2}}$ in a neighborhood of $\Sigma_{Z_{2}} \cup$ $\partial Z_{2}$ which obeys the first two conditions of (1.7) and which has s.quare zero near $\Sigma_{Z_{2}}$. However, Meyer-Vietoris shows that there is an obstruction in $\operatorname{coker}\left(\iota^{*}\right)$ 
to extending this form $\omega_{Z_{2}}$ over $Z_{2}-\Sigma_{Z_{2}}$. (There is no such extension problem for $Z_{1}$ and $Z_{3}$; see Lemma 4.2 in [7].)

There is no argument at present which demonstrates that the $\operatorname{coker}\left(\iota^{*}\right)$ obstruction is zero. But all is not lost because the application of Stokes' theorem in (2.28) of [7] requires less than the vanishing of $\omega_{Z_{2}} \wedge \omega_{Z_{2}}$ near $\Sigma_{Z_{2}}$. The application of Stokes' theorem requires only the vanishing of $\omega_{Z_{2}} \wedge \omega_{Z_{2}} \wedge \omega_{Z_{2}}$.

With this fact understood, remark that $\underline{\omega}_{Z_{2}}-\mu$ has cube zero if $\underline{\omega}_{Z_{2}}$ and $\mu$ both have square zero. Thus, the issue is framed as follows: Can the obstruction in coker $\left(\iota^{*}\right)$ be killed by adding a closed form $\mu$ to $\underline{\omega}_{Z_{2}}$, where $\mu$ has square zero, is smooth near $\Sigma_{Z_{2}}$ and has support on $Z_{2}$ ? As demonstrated in Section 6 , the answer to this question is yes.

The construction of $\mu$ as above requires that care be taken with the construction of $E_{ \pm}$for $\Sigma_{Z_{2}}$. In particular, $H^{2}\left(E_{ \pm}\right)$must be controlled; as well as the kernel of a certain homomorphism

$$
\iota^{\prime}: H_{2}\left(\Sigma_{Z_{2}}\right) \longrightarrow H_{2}\left(Z_{2}-\Sigma_{Z_{2}}\right) .
$$

The control of $\operatorname{ker}\left(\iota^{\prime}\right)$ requires arguments which do not appear in [7].

\section{e) The remaining sections.}

Here is a brief outline of the remainder of this article: Sections 2, 3, 4 and 5 concern themselves with the construction of $E_{ \pm}$for $\Sigma_{Z_{2}}$. In fact, Sections 2 and 3 are occupied with various preliminary constructions on the cobordism $W_{2}$, with the proper introduction of $E_{ \pm}$reserved for Section 5 . Section 4 describes a preliminary version of $E_{ \pm}$, while Section 5 constructs the final version from the preliminary version by ambient surgery in $W_{2}$.

The proof of Theorem 1.3 is completed in Section 6 .

2 Morse Theory. This section serves as a preliminary digression to introduce certain Morse theoretic constructions that are used in the subsequent construction of $E_{ \pm}$and $\Sigma_{Z}$. The subject here is a compact, oriented, 4-dimensional cobordism between a pair of compact, oriented 3-manifolds with the rational homology of $S^{3}$. Given such a cobordism, one can find a second cobordism which is described by (1.4) and Proposition 3.2 in [7]. The whole of the discussion in [7] concentrates on the factors $W_{1}$ and $W_{3}$ (which have the rational homology of $S^{3}$ ); and the discussion here will concentrate on the factor $W_{2}$.

With this last point understood, let $M_{0,1}$ be a pair of compact, oriented 3-manifolds, both with the rational homology of $S^{3}$. In this section, W will denote a compact, oriented, 4-dimensional cobordism from $M_{0}$ to $M_{1}$ which has a good Morse function with only index 2 critical points. (Section 3a in [7] defines a "good" Morse function.)

\section{a) Algebraic considerations.}

The intersection form, $\beta$, for $W$ is a bilinear form on $H_{2}(W ; \mathbb{Z})$ which is non-degenerate and symetric. Suppose that this form is conjugate over $\operatorname{Gl}(\cdot, \mathbb{Z})$ to a form which appears in (1.3). 
Concerning the homology of $W$, remember that $W$ has a good Morse function with only index 2 critical points. The relative homology exact sequence gives

$$
\begin{aligned}
0 \longrightarrow H_{2}(W ; \mathbb{Z}) & \longrightarrow H_{2}\left(W, M_{0} ; \mathbb{Z}\right) \longrightarrow H_{1}\left(M_{0} ; \mathbb{Z}\right) \\
& \longrightarrow H_{1}(W ; \mathbb{Z}) \longrightarrow 0,
\end{aligned}
$$

and the group $H_{2}\left(W, M_{0} ; \mathbb{Z}\right)$ is freely generated. This implies that $H_{2}(W ; \mathbb{Z})$ is freely generated too. Let $\left\{\sigma_{i}\right\}_{i=1}^{r}$ be a given basis for $H_{2}\left(W, M_{0} ; \mathbb{Z}\right)$, and $\left\{\tau_{i}\right\}_{i=1}^{r}$ for $H_{2}(W ; \mathbb{Z})$. Then the image of $\left\{\tau_{i}\right\}$ in $H_{2}\left(W, M_{0} ; \mathbb{Z}\right)$ is given as $\left\{\tau_{j} \equiv \Sigma_{j} S_{i, j} \cdot \sigma_{j}\right\}$ for some integer valued matrix $\left(S_{i, j}\right)$. The matrix $\left(S_{i, j}\right)$ is invertible over $\mathbb{Q}$; it is unimodular if and only if $H_{1}\left(M_{0} ; \mathbb{Z}\right)=0$.

Given the basis $\left\{\tau_{i}\right\}$ for $H_{2}(W ; \mathbb{Z})$, then one can find a basis $\left\{\sigma_{i}\right\}$ for $H_{2}\left(W, M_{0} ; \mathbb{Z}\right)$ for which the matrix $\left(S_{i, j}\right)$ is upper triangular (see, e.g., [5]) with positive diagonal entries. That is,

$$
S_{i, j}=0 \quad \text { if } i>j \text { and } S_{i, i}>0 \text {. }
$$

Note: If $H_{1}\left(M_{0} ; Z\right)=0$, then the basis $\left\{\sigma_{j}\right\} \equiv\left\{\tau_{j}\right\}$ is allowed.

Let $f: M \longrightarrow[0,1]$ be the good Morse function with only index 2 critical points. One can arrange for such an $f$ to have one critical level, $f^{-1}(1 / 2)$. A choice of pseudo-gradient for the function $f$ defines the descending 2-disks, $\left\{B_{p^{-}}: p \in \operatorname{crit}(f)\right\}$, from the critical points of $f$. Each $B_{p^{-}}$is an embedded 2-disks in $f^{-1}([0,1 / 2])$ to which $f$ restricts with a single maximum, $p$. Orient these disks and they give a basis for $H_{2}\left(W, M_{0} ; \mathbb{Z}\right)$.

Using Milnor's basis theorem (Theorem 7.6 in [6]), one can find:

1) A good Morse function $f$ on $W$ with critical value $1 / 2$ and only index 2 critical points.

2) A labeling $\left\{b_{1}, \ldots, b_{r}\right\}$ of $\operatorname{crit}(f)$.

3) A pseudo-gradient, $v$, for $f$. by

And, these are such that the given basis $\left\{\sigma_{i}\right\}_{i=1}^{r}$ for $H_{2}\left(W, M_{0} ; \mathbb{Z}\right)$ is given

$$
\left\{\sigma_{i} \equiv\left[B_{p^{-}}\right]: p=b_{i}\right\}_{i=1}^{r} .
$$

Here, $\left[B_{p^{-}}\right] \in H_{2}\left(W, M_{0} ; \mathbb{Z}\right)$ is the fundamental class for an appropriate choice of orientation for $B_{p^{-}}$.

\section{b) Factoring the cobordism.}

It proves convenient to factor the cobordism $W$ into a linear chain of simpler cobordisms. The following proposition describes the process:

Proposition 2.1. Let $M_{0,1}$ be a pair of compact, oriented 3-manifolds, each with the rational homology of $S^{3}$. Let $W$ be a compact, connected, oriented 4 dimensional cobordism between $M_{0}$ and $M_{1}$. Assume that the intersection 
form for $W$ obeys (1.3) and assume that $W$ has a good Morse function with only index 2 critical points. Then $W$ can be decomposed as

$$
W \equiv \cup_{j=1}^{n} W_{j}
$$

where $W_{j} \subset W$ is a compact 4-dimensional submanifold with two boundary components, $F_{j}$ and $F_{j+1}$, which are embedded, 3-dimensional submanifolds of $W$. These have the following properties:

1) For each $j, F_{j}$ has the rational homology of $S^{3}$.

2) $F_{n+1}=M_{0}$ and $F_{1}=M_{1}$.

3) For each $j, W_{j} \cap W_{j-1}=F_{j}$.

4) For each $j, H_{2}\left(W_{j} ; \mathbb{Z}\right) \approx \oplus_{2} \mathbb{Z}$ and the intersection matrix is conjugate by $G L(2 ; \mathbb{Z})$ to $H\left(m_{j}\right)$ for some $m_{j} \in \mathbb{Z}-\{0\}$.

5) For each $j, W_{j}$ has a good Morse function which has only two critical points, both with index 2. tion.

The remainder of this subsection is occupied with the proof of this proposi-

Proof. The first step is the construction of the $W_{j}$, and the second step verifies their properties.

Step 1: Because of (1.3), the number $r$ of critical points of $f$ must be even. Given this point, fix small $\epsilon>0$ and modify f slightly so that for $j \in\{1, \ldots, r / 2\}$, the critical points $\left(b_{2 j}, b_{2 j-1}\right)$ have critical value $1 / 2-\epsilon \cdot(j / r)$. Thus, $\left(b_{r}, b_{r-1}\right)$ have the smallest critical value, while $\left(b_{2}, b_{1}\right)$ have the largest critical value.

Set $F_{r / 2+1} \equiv M_{0}$, and for $j \in\{2, \ldots, r / 2\}$, let

$$
F_{j} \equiv f^{-1}(1 / 2-\epsilon \cdot(j-1 / 2) / r) .
$$

Note that each $F_{j}$ is a smooth, oriented submanifold which splits $W$ into two pieces. For $2 \leq j \leq r / 2$, let $V_{j} \subset W$ denote the closure of the component of $W-F_{j}$ which contains $M_{0}$.

Set $W_{r / 2} \equiv V_{r / 2}$ and for $1<j<r / 2$, set

$$
W_{j} \equiv V_{j}-\operatorname{int}\left(V_{j}+1\right) \text {. }
$$

For $j=1$, define $W_{1} \equiv W-\operatorname{int}\left(V_{2}\right)$ and define $F_{1} \equiv M_{1}$.

Step 2: Consider now the properties of the $\left\{W_{j}\right\}$ and $\left\{F_{j}\right\}$ : First of all, Assertions 2 and 3 of Proposition 2.1 follow by construction. As for Assertion 5, note that $W_{j}$ is a submanifold with boundary in $W$ which contains no critical points of $f$ on its boundary, and which contains only the critical points $b_{2 j}, b_{2 j-1}$ of $f$ in its interior. Thus, a rescaling of $f$ on $W_{j}$ will yield a good Morse function on $W_{j}$ to verify Assertion 5 of Proposition 2.1.

The proofs of Assertions 1 and 4 of Proposition 2.1 require a digression to construct representative cycles for the generators $\left\{\tau_{i}\right\}$ of $H_{2}(W ; \mathbb{Z})$. The cycle for a given $\tau_{i}$ will be the fundamental class of a submanifold $T_{i} \subset W$.

To start the digression, remember that $H_{2}(W ; \mathbb{Z})$ is assumed to have a basis $\left\{\tau_{j}\right\}_{j=1}^{r}$ in which the intersection form is given by (1.3). And, remember that 
the image of $\tau_{i}$ in $H_{2}\left(W, M_{0} ; \mathbb{Z}\right)$ is given by $\Sigma_{b \in \operatorname{crit}(f)} S_{i, j(b)} \cdot \sigma_{j(b)}$, where $\sigma_{j(b)} \mathrm{s}$ shorthand for $\left[B_{b-}\right]$ with $b \equiv b_{j}$. (This introduces the indexing function $j(\cdot)$ : $\operatorname{crit}(f) \longrightarrow\{1, \ldots, r\}$ which is defined so that $j(b) \equiv j$ when $b \equiv b_{j}$.) Finally, remember that the Morse function $f$ and its pseudo-gradient have been assumed chosen so that the matrix $\left(S_{i, j}\right)$ obeys $(2.2)$.

The submanifold representative $T_{i}$, for $\tau_{i}$ can be recovered from $\left(S_{i, j}\right)$ and $\left\{B_{b-}: b \in \operatorname{crit}(f)\right\}$ by the following construction: Let $M_{1 / 4} \equiv f^{-1}(1 / 4)$. Note that $M_{1 / 4}$ is diffeomorphic to $M_{0}$. Note as well that $C_{b-} \equiv B_{b-} \cap M_{1 / 4}$ is an embedded circle which is naturally oriented given that $B_{b-}$ is oriented. Thus, $C_{b-}$ determines a homology class, $\left[C_{b-}\right] \in H_{1}\left(M_{1 / 4} ; \mathbb{Z}\right)$. Meyer-Vietoris (Eq. (2.1)) implies that

$$
\mu_{i} \equiv \Sigma_{b \in \operatorname{crit}(f)} S_{i, j(b)} \cdot\left[C_{b-}\right]=0 \in H_{1}\left(M_{1 / 4} ; \mathbb{Z}\right)
$$

Construct push-offs of each $C_{b-}$ by taking a push-off copy of the corresponding $B_{b-}$ and intersecting with $M_{1 / 4}$. Let $\underline{\mu}_{i} \subset M_{1 / 4}$ denote the oriented 1dimensional submanifold which is the union, indexed by $b \in \operatorname{crit}(f)$, of $\left|S_{i, j(b)}\right|$ push-off copies of $C_{b-}$, oriented correctly when $S_{i, j(b)}>0$ and oriented incorrectly otherwise. According to (2.8), this $\underline{\mu}_{i}$ bounds an oriented surface with boundary, $R_{i} \subset M_{1 / 4}$, which is such that $\operatorname{int}^{i}\left(R_{i}\right) \cap \underline{\mu}_{i}=\emptyset$.

With $R_{i}$ understood, represent $\tau_{i}$ by the fundamental class of a subvariety $T_{i}^{\prime}$ which is defined to be the union of $R_{i}$ with the union, indexed by $b \in \operatorname{crit}(f)$, of $\left|S_{i, j(b)}\right|$ push-off copies of $B_{b-}$, oriented correctly if $S_{i, j(b)}>0$ and oriented incorrectly otherwise.

Smooth the corners of $T_{i}^{\prime}$ near $\underline{\mu}_{i}$ to obtain an embedded surface, $T_{i} \subset W$. This $T_{i}$ is naturally oriented and its fundamental class represents the class $\tau_{i}$.

End the digression.

To return to the proof of Proposition 2.1, and, in particular, the proof of Assertion 1. By construction, $H_{2}\left(V_{j}, M_{0} ; \mathbb{Z}\right)$ is generated by $\left\{\left[B_{b-}\right]: j(b) \geq 2 j-1\right\}$ and thus is a free group. Since $M_{0}$ is a rational homology sphere, $H_{2}\left(M_{0} ; \mathbb{Z}\right)=0$ and therefore (1.3) (with $V_{j}$ replacing $W$ ) asserts that $H_{2}\left(V_{j} ; \mathbb{Z}\right.$ ) is also free; by construction, its generators are $\left\{\left[T_{i}\right]: i \geq 2 j-1\right\}$. The intersection form of $V_{j}$ is the restriction of the form for $W$ to $\left\{\left[T_{i}\right]: i \geq 2 j-1\right\}$. This is a sum as in (1.3) and is non-degenerate over $\mathbb{Q}$. The non-degeneracy of the intersection form of $V_{j}$ over $\mathbb{Q}$ implies that $F_{j}$ is a rational homology sphere.

To prove Assertion 4 , note that $H_{2}\left(W_{j}, F_{j+1} ; \mathbb{Z}\right)$ is freely generated by $\left\{\left[B_{b-} \cap\right.\right.$ $\left.W_{j}\right]: j(b)=2 j-1$ or $2 j$ \}. Since $F_{j+1}$ is a rational homology sphere, $H_{2}\left(F_{j+1} ; \mathbb{Z}\right)$ $=0$ and so the $\left(W_{j}, F_{j+1}\right)$ analog of $(1.3)$ implies that $H_{2}\left(W_{j} ; \mathbb{Z}\right)$ is free of rank 2.

Furthermore, the intersection form on $H_{2}\left(W_{j} ; \mathbb{Z}\right)$ must be non-degenerate because the boundary of $W_{j}$ has no rational homology. In fact, the inclusion of $W_{j}$ into $V_{j}$ induces an injection $H_{2}\left(W_{j} ; \mathbb{Z}\right) \longrightarrow H_{2}\left(V_{j} ; \mathbb{Z}\right)$ with image the generators $\left[T_{2 j-1}\right]$ and $\left[T_{2 j}\right]$. This implies the statement in Assertion 5 concerning the intersection form on $H_{2}\left(W_{j} ; \mathbb{Z}\right)$.

Here is why $H_{2}\left(W_{j} ; \mathbb{Z}\right)$ injects into $H_{2}\left(V_{j} ; \mathbb{Z}\right)$ : One must prove that the submanifolds $\left\{T_{2 j}, T_{2 j-1}\right\}$ are homologous to submanifolds which lie in $W_{j}$. 
This happens if $T_{2 j}$ and $T_{2 j-1}$ have zero intersection number with all $B_{b-}$ for $b=b_{i}$ and $i>2 j$. Indeed, if $T \equiv T_{2 j}, T_{2 j-1}$ has zero intersection number as described, then the intersection points of $T$ with each such $B_{b-}$ can be paired as \pm pairs. (One point with positive intersection number, and one with negative.) Then, surgery on these embedded $S^{0}$ 's in $T$ will yield a new surface, $T^{\prime}$, (with larger genus) which is homologous to $T$ and which has no intersection with $B_{b-}$ when $b \equiv b_{i}$ and $i>j$. (Mimic the tubing construction in Section $5 \mathrm{~d}$ of [7].) The pseudo-gradient flow can then be used to isotope this $T^{\prime}$ into $W_{j}$.

With the preceding understood, the lemma follows with the realization that the intersection number of $T$, as above, with $B_{b-}$, as above, is a linear functional of the entries of the matrix $\left(S_{i, j}\right)_{i>j}$. And, this is, by assumption, the zero matrix.

c) $Z$ and $W \times W$.

This subsection describes $Z \subset W \times W$, a submanifold with boundary. For the most part, the discussion here mirrors the discussion in Section $3 \mathrm{~g}$, h of [7] where an analogous $Z$ is defined.

The stage is set with the following Definition:

Definition 2.2. Let $M_{0}, M_{1}$ be compact, oriented 3-manifolds with the rational homology of $S^{3}$.

- A simple type cobordism: A cobordism $W$ between $M_{0}$ and $M_{1}$ is of simple type if the following criteria are met:

1) $W$ is oriented and connected.

2) $W$ has a good Morse function with only two critical points, both of index 2.

3) $H_{2}(W ; \mathbb{Z}) \approx \mathbb{Z}^{2}$, and the intersection form of $W$ is conjugate over $G L(2 ; \mathbb{Z})$ to $H(m)$ for some integer $m \neq 0$.

- A simple type Morse function: Let $W$ be a cobordism of simple type. Let

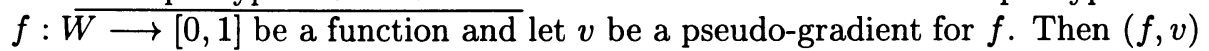
are of simple type if the following criteria are met:

1) $\overline{f_{-1}(0)=M_{0}}$ and $f_{-1}(1)=M_{1}$.

2) $d f \neq 0$ near $\partial W$.

3) $f$ has only two critical points, $\left(b_{1}, b_{2}\right)$, both with index 2 .

4) $15 / 16<f\left(b_{2}\right)<1 / 2<f\left(b_{1}\right)<17 / 16$.

5) There are integers $m_{1}>0, m_{2}>0$ and $m_{1,2}$; and there are orientations of the descending disks from $b_{1}$ and $b_{2}$ such that

$$
\sigma_{1} \equiv m_{1} \cdot\left[B_{b_{1}-}\right]+m_{1,2} \cdot\left[B_{b_{2}-}\right] \text { and } \sigma_{2} \equiv m_{2} \cdot\left[B_{b_{2}-}\right]
$$

generate the image in $\mathrm{H}_{2}\left(W, M_{0} ; \mathbb{Z}\right)$ of $\mathrm{H}_{2}(W ; \mathbb{Z})$.

6) The pseudo-gradient $\mathrm{v}$ is good in the sense of Definition 3.1 in [7].

With the stage set, assume below that $W$ is a cobordism of simple type, and that $(f, v)$ are a pair of Morse function and pseudo-gradient on $W$ which are also of simple type. 
As in Section 3g of [7], introduce

$$
\underline{Z} \equiv\{(x, y) \in W \times W: F(x, y) \equiv f(y)-f(x)=0\} .
$$

Define $Z \subset \underline{Z}$ by intersecting the latter with the compliment in $W \times W$ of (open) small radius balls about $\left(b_{1}, b_{1}\right)$ and $\left(b_{2}, b_{2}\right)$. That is, mimick the constructions in Sections $3 \mathrm{i}$ and $3 \mathrm{~h}$ of [7].

Some properties of $Z$ are listed below:

A manifold: $Z$ is a manifold with boundary,

$$
\partial Z=\left(M_{0} \times M_{0}\right) \cup\left(M_{1} \times M_{1}\right) \cup\left(S^{3} \times S^{3}\right)_{b_{1}} \cup\left(S^{3} \times S^{3}\right)_{b_{2}} ;
$$

here $\left(S^{3} \times S^{3}\right)_{b}$ is the link around $Z$ 's singularity at $(b, b)$. (See Section $3 \mathrm{~h}$ of [7].)

Orientation: The manifold $\operatorname{int}(Z)$ is naturally oriented using the orientation from $W \times W$ along with $d F$ to trivialize the normal bundle to int $(Z)$ in $W \times W$. Orient the various components of (2.11) as described in Section $3 \mathrm{~h}$ of [7].

Homology: The rational homology of $Z$ is described by

LemMa 2.3. Let $W$ be as described above. Then the following hold:

1) $H_{0}(Z) \approx R$.

2) $H_{1}(Z) \approx 0$.

3) The inclusion $Z \subset W \times W$ induces $H_{2}(Z) \approx H_{2}(W \times W) \approx \mathbb{R}^{4}$.

4) There is a surjection

$$
0 \longleftarrow H_{3}(Z) \longleftarrow L_{-} \oplus L_{+} \oplus H_{3}(\partial Z) \text {. }
$$

Here, $L_{ \pm} \approx \mathbb{R}$ are freely generated by embedded 3-spheres in $Z$ as described in Equations (3.32) and (3.33) of [7].

Proof. Mimic the proof of Lemma 3.7 in [7].

3 Constructing $T_{1}$ and $T_{2}$. The constructions in [7] aside, the proof of Theorem 1.3 is mostly occupied with constructions on $W_{2} \times W_{2}$, where $W_{2}$ is described in (1.4). The previous subsection introduced a factorization of such a $W_{2}$ as a sequence of cobordisms of simple type, each with a Morse function $f$ and pseudo-gradient $v$ of simple type. (See Proposition 2.1 and Definition 2.2.) The required constructions for $W_{2}$ in (1.4) can be reduced to a series of identical constructions, one on each simple type cobordism factor in (2.5).

With the preceding as motivation, this section will restrict attention to a cobordism $W$ of simple type with a Morse function $f$ and pseudo-gradient $v$ which are of simple type also. The purpose of this section is to describe a very useful pair of 2-dimensional submanifolds of $W, T_{1}$ and $T_{2}$, whose fundamental classes generate $H_{2}(W)$ and give the intersection form $H(m)$. Thus, this section serves as a second digression before the construction of $E_{ \pm}$. 


\section{a) Reconstructing $T_{2}$.}

The submanifold $T_{2}$ is obtained by smoothing the corners of a $C^{0}$ embedding of a smooth surface into $W$. This embedding can be obtained as follows:

Step 1: Let $V \subset W$ denote the set $\{x \in W: f(x) \geq 1 / 4\}$. To construct $T_{2}$, first introduce the number $m_{2}$ from (2.9) and take $m_{2}$ push-off copies of $B_{b_{2}}-\cap V$, all with the same orientation. Make these push-offs so that $\mathrm{f}$ restricts to each copy with only one critical point, a maximum. And require that said maximum be close to $b_{2}$ in the following sense: Use the Morse coordinates of (3.2) in [7] and the Euclidean metric on $\mathbb{R}^{4}$ to measure distance. With this understood, the distance from each such minimum to $b_{2}$ should be much less than the number $r$ which is used in (3.29) of [7] to define the boundary of $Z$.

To be precise, work in the Morse coordinates of (3.2) in [7] near $b_{2}$. Choose $m_{2}$ distinct unit vectors $\left\{n_{\alpha}\right\}$ in the $\left(x_{3}, x_{4}\right)$ plane. Then, choose $\epsilon>0$ but with $\epsilon<<r$. Define the $\alpha$ 'th push-off of $B_{b_{2}}$ - to be the set

$$
\left\{\left(x_{1}, x_{2}, x_{3}, x_{4}\right):\left(x_{3}, x_{4}\right)=\epsilon \cdot n_{\alpha}\right\}
$$

Step 2: Use $B_{b_{2}-}^{\prime}$ to denote the resulting $m_{2}$ push-offs of $B_{b_{2}}$; this is an oriented, submanifold with boundary in $V$. It is important to realize that $\partial B_{b_{2}-}^{\prime} \subset M_{1 / 4} \equiv f^{-1}(1 / 4)$ is a disjoint union of $m_{2}$ embedded, oriented circles. These circles bound an oriented, embedded surface with boundary $R_{2} \subset M_{1 / 4}$ which intersects $\partial B_{b_{2}-}^{\prime}$ as $\partial R_{2}$. Take such an $R_{2}$ which is connected and which has no compact components.

Set

$$
T_{2}^{\prime} \equiv B_{b_{2}-}^{\prime} \cup R_{2}
$$

This is a (tame) $C^{0}$-embedding of a smooth surface; the embedding is smooth save for the corners along $\partial R_{2}$. However, these corners are right angle corners in a suitable coordinate system and can be smoothed without difficulty. The resulting smooth submanifold of $W$ is $T_{2}$.

Step 3: The push-offs $B_{b_{2}-}^{\prime}$ can be constructed so that $T_{2}^{\prime}$ has the following properties:

1) No pseudo-gradient flow line intersects $T_{2}^{\prime}$ more than once.

2) No pseudo-gradient flow line is anywhere tangent to $B_{b_{2}-}^{\prime}$.

2) $T_{2}^{\prime}$ has empty intersection with $B_{b_{2}-}$.

(3.3) 3) The restriction of $f$ to $B_{b_{2}-}^{\prime}$ has only index 2 critical points, and precisely one on each component.

4) Each component of $B_{b_{2}-}^{\prime}$ intersects $B_{b_{2}}+$ transverally in exactly one point.

To satisfy (3.3), first note that the explicit description in (3.2) for $B_{b_{2}-}^{\prime}$ obeys (3.3). (This is because the vectors $\left\{n_{\alpha}\right\}$ in (3.1) are assumed to be distinct.) 
Second, note that $B_{b_{2}-}^{\prime}$ can be made so that: (3.3) holds, $\partial B_{b_{2}-}^{\prime}$ lies on the boundary of an embedded solid torus $N \subset M_{1 / 4}$, and past $\left(B_{b_{1}-}^{\prime}\right) \cap M_{1 / 4}$ lies in the interior of $N$. Note that the core circle of $N$ is $B_{b_{2}-} \cap M_{1 / 4}$. (Recall from Section 5a in [7] the definition of the past and future of a set $U$ (written past $(U)$ and fut $(U)$, respectively). For example, past $(U) \subset W$ is the set of points which can be obtained from $U$ by traveling along pseudo-gradient flow lines to decrease f.) The Morse coordinates in (3.1) extend over a neighborhood of $B_{b_{2}-}$ in $W$, and with this understood, the tubular neighborhood $N$ is described by

$$
N \approx\left(x_{1}, x_{2}, x_{3}, x_{4}\right): x_{3}^{2}+x_{4}^{2} \leq \epsilon \text { and } x_{1}^{2}+x_{2}^{2}=x_{3}^{2}+x_{4}^{2}+c,
$$

Here $c>0$ is an appropriate constant.

Equation (3.3) follows by showing that $\partial B_{b_{2}-}^{\prime}$ bounds an embedded surface with boundary in the compliment of $\operatorname{int}(N)$. And, such a surface exists because the class $\tau_{2} \in H_{2}(W ; \mathbb{Z})$ has zero self intersection number.

With the coordinates of (3.1) and (3.4) understood, the submanifold $R_{2}$ can be assumed to intersect a neighborhood of $N$ as the set of $\left(x_{1}, x_{2}, x_{3}, x_{4}\right)$ which obey:

1) $\left(x_{3}, x_{4}\right)=t \cdot n_{\alpha}$ for $t \geq \epsilon$ and some $\alpha \in\left\{1, \ldots, m_{2}\right\}$.

2) $x_{1}^{2}+x_{2}^{2}=x_{3}^{2}+x_{4}^{2}+c$.

Step 4: Henceforth, assume that $T_{2}^{\prime}$ in (3.2) obeys (3.3) and (3.5). The corner in $T_{2}^{\prime}$ at $\partial R_{2}$ can be smoothed to produce a smooth submanifold $T_{2} \subset$ $f^{-1}([1 / 4,1])$ which contains $R_{2}$ as a submanifold. The manifold $T_{2}$ is obtained by flowing points in $B_{b_{2}-}^{\prime}$ near $M_{1 / 4}$ slightly into their past so that the result (also called $B_{b_{2}-}^{\prime}$ ) is tangent to $M_{1 / 4}$ at $\partial B_{b_{2}-}^{\prime}$ to infinite order. Note that $T_{2}$ can be so constructed to obey

1) No pseudo-gradient flow line intersects $T_{2}$ more than once.

2) No pseudo-gradient flow line is anywhere tangent to $T_{2}$.

(3.6) 3) $T_{2}$ has empty intersection with $B_{b_{2}-}$.

4) Where $f>1 / 4+1 / 100$, the restriction of $f$ to $T_{2}$ has only index 2 critical points.

\section{b) Constructing $T_{1}^{\prime}$.}

It is convenient to replace $T_{1}$ of the previous section with a different, though homologous submanifold.

The construction of the new version of $T_{1}$ requires first the construction of a piece-wise smooth submanifold $T_{1}^{\prime} \subset W$ which is defined in this subsection. Second, the construction of the new $T_{1}$ requires a modification of $T_{1}^{\prime}$ to give a smooth submanifold, $T_{1}^{\prime \prime} \subset W$. This $T_{1}^{\prime \prime}$ is described in the next subsection. The new version of $T_{1}$ is finally presented in Subsection $2 \mathrm{f}$, below.

The construction of $T_{1}^{\prime}$ is accomplished in the following steps:

Step 1: Let $M_{3 / 8} \equiv f^{-1}(3 / 8)$. Introduce the integers $m_{1}$ and $m_{1,2}$ from (2.9). Use $B_{b_{1}-}^{\prime}$ to denote the union of $m_{1}$ disjoint, push-off copies of $B_{b_{1}} \cap$ 
fut $\left(M_{3 / 8}\right)$ together with $\left|m_{1,2}\right|$ disjoint, push-off copies of $B_{b_{2}}-\cap \operatorname{fut}\left(M_{3 / 8}\right)$.

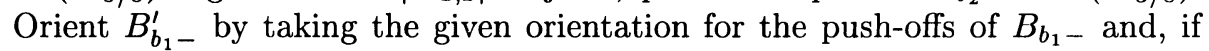
$m_{1,2}>0$, the given orientation for the push-offs of $B_{b_{2}-}$. However, if $m_{1,2}<0$, use the opposite orientation for the push-offs of $B_{b_{2}-}$.

The function $\mathrm{f}$ should restrict to each of the $m_{1}$ push-off copies of $B_{b_{1}-}$ to have only one critical point, a maximum. And this maximum should be close to $b_{1}$; its distance should be much less than the number $r$ from (3.29) in [7] when distance is measured using the Euclidean metric on $\mathbb{R}^{4}$ in the Morse coordinates from (3.2) of [7]. Thus, use the Morse coordinates around $b_{1}$ and (3.1) to define the typical push-off of $B_{b_{1}-}$; in (3.1), use $\epsilon<<r$ and use distinct $\left\{n_{\alpha}\right\}$.

Note that fut $\left(T_{2}\right)$ intersects $B_{b_{1}}$ - in a finite set of arcs with one endpoint at $b_{1}$. With this understood, make the push-off copies of $B_{b_{1}} \cap$ fut $\left(M_{3 / 8}\right)$ which comprise $B_{b_{1}-}^{\prime}$ such that each intersects $B_{b_{1}}+$ transversally in a single point, and such that each intersects fut $\left(T_{2}\right)$ as a finite set of half-open arc with the following properties:

1) Each end-point lies on $\partial B_{b_{1}-}^{\prime}$.

2) The closure of each arc has its second endpoint on the intersection with $B_{b_{1}+}$.

3) The function $f$ restricts to each arc without critical points.

Meanwhile, the function $\mathrm{f}$ should restrict to each of the $\left|m_{1,2}\right|$ copies of $B_{b_{2}-}$ to have only one critical point, a maximum. In this case, this maximum should have distance from $b_{2}$ much greater than $r$ (of (3.29) in [7]) when measured with the Euclidean metric in the Morse coordinates of (3.2) in [7]. Thus, a push-off copy of $B_{b_{2}-}$ in $B_{b_{1}-}^{\prime}$ should be given by (3.1) but with $\epsilon>>r$. Also, for these $\left|m_{1,2}\right|$ push-offs of $B_{b_{2}-}$, use a set of unit vectors $\left\{n_{\alpha}^{\prime}\right\}$ in (3.2) that is disjoint from the set that was used to define $B_{b_{2}-}^{\prime}$.

Require that

$$
\operatorname{fut}\left(T_{2}\right) \cap B=\emptyset
$$

whenever $B \subset B_{b_{1}-}^{\prime}$ is any of the set of $\left|m_{1,2}\right|$ push-off copies of $B_{b_{2}} \cap \operatorname{fut}\left(M_{3 / 8}\right)$ in $B_{b_{1}-}^{\prime}$. (See (3.5) and (3.6.3).)

Step 3: The boundary of $B_{b_{1}-}^{\prime}$ is a disjoint union of oriented circles in $M_{3 / 8}$. This union of circles defines a cycle which is null homologous in $M_{3 / 8}$. And, $\partial B_{b_{1}-}^{\prime}$ bounds an oriented, embedded surface with boundary, $R_{1}^{\prime} \subset M_{3 / 8}$, which intersects $\partial B_{b_{1}-}^{\prime}$ as $\partial R_{1}^{\prime}$. Take $R_{1}^{\prime}$ which is connected and which has no compact components.

With $R_{1}^{\prime}$ and $B_{b_{1}-}^{\prime}$ understood, set

$$
T_{1}^{\prime} \equiv B_{b_{1}-}^{\prime} \cup R_{1}^{\prime}
$$


c) Constructing $T_{1}^{\prime \prime}$.

Given that $T_{2}$ has already been constructed, it is desirable to modify $T_{1}^{\prime}$ by isotopy so that the result, $T_{1}^{\prime \prime}$, has the following property:

1) No pseudo-gradient flow line intersects $T_{1}^{\prime \prime}$ more than once where $f \leq \sup \left(f \mid T_{2}\right)$.

2) No pseudo-gradient flow line is anywhere tangent to $T_{1}^{\prime \prime}$ where $f \leq \sup \left(f \mid T_{2}\right)$.

If $T_{1}^{\prime \prime} \equiv T_{1}^{\prime}$ is given by (3.9), this condition may not hold. (Note that (3.10) holds separately for $R_{1}^{\prime}$ and for $B_{b_{1}}^{\prime}$.) However, (3.10) can always be achieved by redefining the push-offs in $B_{b_{1}}^{\prime}$ - .

The redefinition of $B_{b_{1}}^{\prime}$ - begins with the following observation: A component $B \subset B_{b_{1}-}^{\prime}$ which is a push-off copy of $B_{b_{2}-}$ needs a choice of $\epsilon$ in (3.1) for its definition. Let $\epsilon_{1}$ denote the chosen value of $\epsilon$. Likewise, each copy of $B_{b_{2}-}$ comprising $B_{b_{2}-}^{\prime}$ needs a choice of $\epsilon$ in (3.1). Use $\epsilon_{2}$ to denote the choice here. One is required to choose $\epsilon_{1}>>\epsilon_{2}$. If $\epsilon_{1}$ is, none-the-less, much less than $f\left(b_{1}\right)-f\left(b_{2}\right)$, then there are numbers $h_{1}<h_{2}$ which are both greater than $\sup \left(f \mid T_{2}\right)$ but which are both significantly less than $f\left(b_{1}\right)$ (as measured in multiples of $\epsilon_{1}$ ) and also less than the maximum of $f$ on any of the components of $B_{b_{1}-}^{\prime}$.

Note next that $\partial B_{1-}^{\prime}$ is disjoint from the solid torus $N$ in (3.4). This implies that $\partial R_{1}^{\prime}$ has a collar $C \subset R_{1}^{\prime}$ which is disjoint from $N$. Each component of $C$ is an embedding in $R_{1}^{\prime}$ of $[0,1] \times S^{1}$; and the convention will be that $\{0\} \times S^{1}$ corresponds to a component of $\partial R_{1}^{\prime}$. Fix such a collar with the following properties: A component which intersects a push-off of $B_{b_{2}-}$ in $B_{b_{2}-}^{\prime}$ should be disjoint from fut $\left(T_{2}\right)$. (See (3.8).) And, a component which intersects a push-off of $B_{b_{1}-}$ in $B_{b_{2}-}^{\prime}$ should intersect fut $\left(T_{2}\right)$ as an arc between said components two boundaries. (See (3.7).)

Define a re-imbedding of $C \cup B_{b_{1}-}^{\prime}$ in $W$ as follows: Move points of $C \cup B_{b_{1}-}^{\prime}$ by an isotopy of $W$ which pushes points along pseudo-gradient flow lines. The result of the isotopy should push $B_{b_{1}}^{\prime}$ to where $f \geq h_{1}$ but it should leave the image of $C$ where $f \leq h_{1}$. Thus, $\partial R_{1}^{\prime}$ is pushed to $f^{-1}\left(h_{1}\right)$. The isotopy should keep fixed the compliment in $C$ of a neighborhood of $\partial R_{1}^{\prime}$ and it should fix points in $B_{b_{1}-}^{\prime}$ where $f \geq h_{2}$. It is not difficult to make this re-imbedding so that

1) The restriction of $f$ to the image of $C$ has no critical points where $f>3 / 8$.

2) The restriction of $f$ to the image of $B_{b_{1}}^{\prime}$ has only index 2 critical points, and there is precisely one on each component.

The embedded image of $C \cup B_{b_{1}}^{\prime}$ gives a piecewise smooth embedding in $W$ of a union of disks. Indeed, the embedded image of $C \cup B_{b_{1}-}^{\prime}$ has a corner where the images of $C$ and of $B_{b_{1}}^{\prime}$ intersect, that is, along $f^{-1}\left(h_{1}\right)$. Choose 
in advance a neighborhood of this corner, and the image of $C \cup B_{b_{1}-}^{\prime}$ can be smoothed in the chosen neighborhood so that the result, $B_{b_{1}-}^{\prime \prime}$, has the following properties:

1) $B_{b_{1}-}^{\prime \prime}=B_{b_{1}-}^{\prime}$ where $f<h_{2}$.

2) $\quad B_{b_{1}-}^{\prime \prime}$ agrees with the image of $C$ where $f \geq h_{1}$.

3) The restriction of $f$ to $B_{b_{1}-}^{\prime \prime}$ has only index 2 critical points where $f>3 / 8$; and there is precisely one on each component.

4) Each component of $B_{b_{1}-}^{\prime \prime}$ is either a push-off copy of $B_{b_{1}-}$, or else one of $B_{b_{2}-}$.

5) A component of $B_{b_{1}-}^{\prime \prime}$ - which is a copy of $B_{b_{1}}$ - intersects $B_{b_{1}+}$ transversely in a single point. Such a component also intersects fut $\left(T_{2}\right)$ in a finite set of halfopen arcs with their boundaries on $M_{3 / 8}$. The closures of each half-open arc is an embedded arc whose other end-point is the intersection point with $B_{b_{1}+}$. Furthermore, $f$ restricts to each half-open arc with no critical points where $f<3 / 8$.

6) A component of $B_{b_{1}-}^{\prime \prime}$ which is a copy of $B_{b_{2}-}$ has empty intersection with fut $\left(T_{2}\right)$.

7) No pseudo-gradient flow line is anywhere tangent to $B_{b_{1}-}^{\prime \prime}$.

Let $R_{1}^{\prime \prime} \equiv R_{1}^{\prime}-C$ and define

$$
T_{1}^{\prime \prime} \equiv R_{1}^{\prime \prime} \cup B_{b_{1}-}^{\prime \prime} .
$$

This submanifold obeys (3.10). (Where $f \geq h_{1}, T_{1}^{\prime \prime}$ is obtained from $R_{1}^{\prime}$ by flowing the latter along pseudo-gradient flow lines.)

\section{d) Intersection of fut $\left(T_{1}^{\prime \prime}\right)$ with $T_{2}$ and $T_{1}^{\prime \prime}$ with fut $\left(T_{2}\right)$.}

The intersection between fut $\left(T_{1}^{\prime \prime}\right) \cap T_{2}$ is the union of a finite set of half-open arcs each of which has its endpoint at one of the points of $T_{1}^{\prime \prime} \cap T_{2}$, and viceversa. (Note that $T_{1}^{\prime \prime} \cap T_{2}=R_{1}^{\prime \prime} \cap T_{2}$.) The closure of each half open arc is an embedded arc with its other end point where $B_{b_{2}-}^{\prime}$ intersects $B_{b_{2}+}$. There are at least $m$ such arcs.

The intersection of $T_{1}^{\prime \prime}$ with fut $\left(T_{2}\right)$ is more complicated. After perturbing $T_{1}^{\prime \prime}$ slightly, this intersection can be assumed to have the following form: It consists of a finite, disjoint set of closed arcs, half-open arcs, and open $\operatorname{arcs}$ in $T_{1}^{\prime \prime}$; and disjoint from these arcs, there is a finite set of disjoint, embedded circles. Each point of $T_{1}^{\prime \prime} \cap T_{2}\left(=R_{1}^{\prime \prime} \cap B_{b_{2}-}^{\prime}\right)$ will be a boundary component for some arc, either half open or closed. (But, there may be more or less arcs than boundary components of arcs.)

The closure of a half-open arc will be a smooth arc whose other endpoint lies on $B_{b_{1}-}^{\prime \prime} \cap B_{b_{1}+}$ (and thus in a push-off copy of $B_{b_{1}-}$ in $B_{b_{1}-}^{\prime \prime}$ ). The closure 
of an open arc will also be a smooth arc, but with both of its endpoints in $B_{b_{1}-}^{\prime \prime} \cap B_{b_{1}+}$. To see that such is the case, introduce $\underline{R}_{2} \equiv$ fut $\left(R_{2}\right) \cap f^{-1}(7 / 16)$. This will intersect $T_{1}^{\prime \prime}$ in the $m_{2}$ push-off copies of $B_{b_{1}-}$. (In fact, its intersection number with the union of said $m_{2}$ copies is equal to $m$. See (5) and (6) of (3.12).) Each intersection point of $\underline{R}_{2}$ with $T_{1}^{\prime \prime}$ has one half-open arc component or one open arc component of $T_{1}^{\prime \prime} \cap$ fut $\left(T_{2}\right)$ passing through it. Furthermore, each half-open arc component intersects precisely one point of $T_{1}^{\prime \prime} \cap \underline{R}_{2}$, while each open arc component intersects precisely two such points. Each half-open arc component intersects $f^{-1}([7 / 16,1])$ as a push-off copy of a pseudo-gradient flow line for $f$ in $B_{b_{1}}$ - which ends in $b_{2}$; and each open arc component intersects $f^{-1}([7 / 16,1])$ in a pair of such push-offs.

The circles in $T_{1}^{\prime \prime} \cap$ fut $\left(T_{2}\right)$ can be assumed to lie in the interior of $R_{1}^{\prime \prime}$. (See (5) and (6) of (3.12).)

It is important to note that there are at least $\mathrm{m}$ half-open arcs components of $T_{1}^{\prime \prime} \cap$ fut $\left(T_{2}\right)$; any less would be incompatible with the assumed value of $m$ for $T_{1}^{\prime \prime} \cdot T_{2}$. If a pair of points in $T_{1}^{\prime \prime} \cap R_{2}$ are points on the same open arc, then these points will have opposite local intersection numbers for $T_{1}^{\prime \prime} \cap T_{2}$.

A similar argument shows that for at least $m$ of these arcs, both the intersection point in $B_{b_{1}-}^{\prime \prime} \cap \underline{R}_{2}$ and the endpoint in $R_{1}^{\prime \prime} \cap B_{b_{2}-}^{\prime}$ are points of positive local intersection number for $T_{1}^{\prime \prime} \cap \underline{R}_{2}$ and for $R_{1}^{\prime \prime} \cap T_{2}$, respectively.

With the preceding understood, fix one half-open arc,

$$
v^{0} \subset\left(T_{1}^{\prime \prime} \cap \operatorname{fut}\left(T_{2}\right)\right),
$$

which intersects $B_{b_{1}-}^{\prime \prime} \cap R_{2}$ at a point of positive local intersection number, and which ends in $R_{1}^{\prime \prime} \cap B_{b_{2}-}^{\prime}$ at a point with positive local intersection number.

\section{e) Homology of $T_{1}^{\prime}$ and $T_{2}$ and the linking matrix.}

There is one additional constraint that must be imposed on $T_{1}^{\prime \prime}$; and this one also requires advanced knowledge of $T_{2}$. Suppose that $T_{1}^{\prime \prime}$ and $T_{2}$ have already been constructed.

The surface $T_{2}$ has some genus $g_{2} \geq 0$. As such, its first homology has a basis which is represented by the fundamental class of a set, $\left\{\eta_{2 \beta}\right\}_{\beta=1}^{2 g_{2}} \subset \operatorname{int}\left(R_{2}\right)$, of $2 \cdot g_{2}$ embedded, oriented circles. Take $n_{1}$ (from above) like oriented, push-off (in $R_{2}$ ) copies of each $\eta_{2 \alpha}$. Together, these form a set $\left\{\rho_{\beta_{i}}^{\prime}\right\}$, where $\beta$ runs from 1 to $2 g_{2}$, and where $i$ runs from 1 to $n_{1}$.

The pseudo-gradient flow pushes $R_{2}$ isotopically into $M_{3 / 8}$ as the submanifold fut $\left(R_{2}\right) \cap M_{3 / 8}$, and thus the circles $\left\{\rho_{\beta_{i}}^{\prime}\right\}$ are pushed isotopically into $M_{3 / 8}$ as a set, $\left\{\rho_{\beta_{i}}\right\} \subset M_{3 / 8}$, of $2 \cdot n_{1} \cdot g_{2}$ circles.

Fix the set of circles $\left\{\rho_{\beta_{i}}\right\}$ once and for all. These circles will be used to constrain $R_{1}^{\prime \prime}$; but a short digression is needed to define these new constraints.

Start the digression by observing that the surface $T_{1}^{\prime \prime}$ has some genus $g_{1} \geq 0$ and so its first homology is represented by the fundamental class of a set of $2 \cdot g_{1}$ embedded, oriented circles, $\left\{\eta_{1 \alpha}\right\}_{\alpha=1}^{2 g_{1}} \subset \operatorname{int}\left(R_{1}^{\prime \prime}\right) \subset M_{3 / 8}$. These generators should be chosen to be disjoint from the arc $v^{0}$ which is described in (3.14). (This is possible because $v^{0}$ is an arc with one endpoint on $\partial R_{1}^{\prime \prime}$ and the other in the interior of $R_{1}^{\prime \prime}$.) 
The manifold $M_{3 / 8}$, being diffeomorphic to $M_{0}$, has the rational homology of $S^{3}$. This means, in particular, that some number $n_{1} \geq 1$ of like oriented pushoff copies (in $R_{1}^{\prime \prime}$ ) of each $\eta_{1 \alpha}$ bounds an embedded surface with boundary, $S_{\alpha} \subset M_{3 / 8}$.

No generality is lost by assuming that $R_{1}^{\prime \prime}$ intersect each of the circles $\left\{\rho_{\beta_{i}}\right\}$ transversally. Likewise, there is no generality lost here by requiring that the $\left\{\eta_{1 \alpha}\right\}$ which generate $H_{1}\left(T_{1}^{\prime \prime}\right)$ be disjoint from the set $\left\{\rho_{\beta_{i}}\right\}$.

Push-off, in $R_{1}^{\prime \prime}$, the $n_{1}$ copies of each $\eta_{1 \alpha}$. Make these close to $\eta_{1 \alpha}$ to insure that the push-off isotopy is disjoint from $\left\{\rho_{\beta_{i}}\right\}$. Find the submanifold with boundary $S_{\alpha} \subset M_{3 / 8}$ which intersects the $n_{1}$ push-off copies of $\eta_{1 \alpha}$ as its boundary.

In general position, each such $S_{\alpha}$ will intersect each of the circles $\rho_{\beta_{i}}$ transversally. So, there is a $2 g_{1} \times 2 g_{2}$ matrix $A \equiv\left(A_{\alpha, \beta}\right)$ where $A_{\alpha, \beta}$ is the sum of the intersection numbers between the surface $S_{\alpha}$ and the $n_{1}$ circles $\left\{\rho_{\beta_{i}}\right\}_{i=1}^{n_{1}}$. (Here, the index $\beta$ is fixed.) The matrix $A$ will be called the linking matrix between the set $\left\{\eta_{1 \alpha}\right\}$ and the set $\left\{\rho_{\beta}\right.$. . Note that the entries $A_{\alpha, \beta}$ are divisible by the integer $n_{1}$, and that the definition of $A_{\alpha, \beta}$ requires the apriori choice of push-offs $\left\{\rho_{\beta_{i}}^{\prime}\right\}$ of $\left\{\eta_{2 \beta}\right\}$.

With the preceding understood, the point of this subsection is to remark that there is an isotopy of $R_{1}^{\prime \prime}$ in $M_{3 / 8}$ ( rel $\partial R_{1}^{\prime \prime}$, the $\operatorname{arc} v^{0}$, and $R_{1}^{\prime \prime} \cap T_{2}$ ) to a surface $R *_{1} \subset M_{3 / 8}$ so that the linking matrix $A^{*}$ between the isotoped circles, $\left\{\eta *_{1 \alpha}\right\}$, and $\left\{\rho_{\beta}\right.$. $\}$ has all entries zero. In fact, this can be accomplished using finger moves to isotope $\eta_{1 \alpha}$ to change its linking number with each $\rho_{\beta 1}$ but leave unchanged the linking number with each $\rho_{\beta_{i}>1}$. (Note that the linking number with $\rho_{\beta_{1}}$ can be changed only by multiples of an integer which divides $n_{1}$, while the entries of the matrix $A_{\alpha, \beta}$ are divisible by $n_{1}$.)

Each such finger move changes $R_{1}^{\prime \prime}$ by an ambient isotopy which fixes the compliment of a small ball in $R_{1}^{\prime \prime}$ and which stretches the interior of this ball over a regular neighborhood of some arc in $M_{3 / 8}$. The ability to simultaneously change all entries of $A$ to zero is based on the fact that the finger move isotopy moves $R_{1}^{\prime \prime}$ only in tubular neighborhoods of arcs.

Because each finger move changes $R_{1}^{\prime \prime}$ only in the neighborhood of a point, these finger can be made away from $\partial R_{1}^{\prime \prime}$, the path $v^{0}$. For the same reason, the finger moves can be done so as to leave $R_{1}^{\prime \prime} \cap T_{2}$ unchanged.

With the preceding understood, it will be assumed in the sequel that there exist $n_{1} \geq 0$ and a set of:

1) circles $\left\{\eta_{2 \beta}\right\} \subset T_{2}$ which generate $H_{1}\left(T_{2}\right)$

for the homology of $T_{2}$,

2) $n_{1}$ push-off copies, $\left\{\left\{\rho_{\beta_{i}}\right\}_{i=1}^{n_{1}}\right\}$, of $\left\{\eta_{2 \beta}\right\}$,

3) circles $\left\{\eta_{1 \alpha}\right\}$ which generate $H_{1}\left(T_{1}^{\prime \prime}\right)$,

with the property that the resulting linking matrix $A=\left(A_{\alpha, \beta}\right)$ has all entries zero. Furthermore, $\left\{\eta_{1 \alpha}\right\}$ will be assumed disjoint from $v^{0}$ of (2.14) and from $\operatorname{fut}\left(\left\{\rho_{\beta, i}^{\prime}\right\}\right)$. 


\section{f) Definition of $T_{1}$.}

With $T_{1}^{\prime \prime}$ understood, the surface $T_{1} \subset W$ can now be constructed by isotoping $T_{1}^{\prime \prime}$ into the future a small amount along pseudo-gradient flow lines.

This construction of $T_{1}$ is accomplished by the following steps:

Step 1: Find an embedding

$$
\varphi: f^{-1}([3 / 8,1]) \longrightarrow f^{-1}([3 / 8,1])
$$

with the following properties:

1) $\varphi$ is the end of an isotopy which moves points along pseudo-gradient flow lines.

2) $\varphi$ is the identity where $f \geq 3 / 8+1 / 100$.

(3.17) 3) Let $\underline{M} \equiv \varphi\left(M_{3 / 8}\right)$. Then $f \mid \underline{M}>3 / 8$.

4) $f$ restricts to $\varphi\left(v^{0}\right)$ with out critical points.

5) $\inf \left(f \mid \varphi\left(\left\{\eta_{1 \alpha}\right\}\right)\right)>\sup \left(f \mid \varphi\left(\left\{\rho_{\beta_{i}} \cap T_{1}^{\prime \prime}\right\}\right)\right)$.

6) $f$ restricts to $\varphi\left(B_{b_{1}-}^{\prime \prime}\right)$ with only index 2 critical points, one on each component.

To find such a $\varphi$, use the pseudo-gradient flow to construct a diffeomorphism

$$
f^{-1}([3 / 8,7 / 16]) \approx M_{3 / 8} \times[3 / 8,7 / 16]
$$

where the pseudo-gradient flow lines are mapped to the lines $p \times[3 / 8,7 / 16]$, and where $f$ is given by projection onto the second factor. With respect to (3.18), the embedding $\varphi$ sends $(p, t)$ to $(p, g(p, t))$, where $g$ is a smooth function. It is left to the reader to find $g$ which makes (3.17) true. (Remark here that $\left\{\eta_{1 \alpha}\right\}$ are disjoint from $v^{0}$ and from $\left\{\rho_{\beta_{i}}\right\}$.

Step 2: With $\varphi$ understood, define

$$
T_{1} \equiv \varphi\left(T_{1}^{\prime \prime}\right)
$$

Also, introduce $R_{1} \equiv \varphi\left(R_{1}^{\prime \prime}\right)$. Here are some important properties of $T_{1}$ : 
1) No pseudo-gradient flow line intersects $T_{1}$ more than once where $f \leq \sup \left(f \mid T_{2}\right)$.

2) No pseudo-gradient is anywhere tangent to $T_{1}$ where $f \leq \sup \left(f \mid T_{2}\right)$.

3) $T_{1} \cap T_{2}=\operatorname{int}\left(R_{1}\right) \cap B_{b_{2}-}^{\prime}$.

4) Where $f \geq 3 / 8+1 / 100$, the restriction of $f$ to $T_{1}$ has only index 2 critical points.

4 A start at $\Sigma_{Z}$. This section begins the construction of the subvariety $\Sigma_{Z_{2}}$ as in (1.6). The plan is to factor the cobordism $W_{2}$ from (1.4) as a sequence of cobordisms of simple type (Definition 2.2), and to define a $\Sigma$. for each component, simple type cobordism in this factorization. Then, $\Sigma_{Z_{2}}$ in (1.6) is defined to be the union of these $\Sigma$. for the constituent simple type cobordisms which comprise $W_{2}$.

With the preceding understood, assume in this section and in Section 5 that $W$, and the Morse function $\mathrm{f}$ and the pseudo-gradient $v$ are of simple type, as defined in Definition 2.2. Use the definitions in Section 2c to define $Z \subset W \times W$. Sections 4 and 5 will construct a particular oriented, dimension-4 subvariety with boundary $\Sigma_{Z} \subset Z$. The boundary of $\Sigma_{Z}$ will sit in $\partial Z$. Furthermore, $\Sigma_{Z}$ will contain a class $\sigma_{Z} \in H_{4}\left(\Sigma_{Z}, \partial \Sigma\right)$ which obeys the conclusions of Lemma 4.1 in [7]. As in Section 4c and (4.10) of [7], the variety $\Sigma_{Z}$ will be given as a union

$$
\Sigma_{Z}=\Delta_{Z} \cup E_{L} \cup E_{R} \cup E_{-} \cup E_{+} .
$$

Here, $\Delta_{Z}$ is as described in Section $4 \mathrm{~d}$ of [7], and $E_{L, R}$ are as described in Section $4 \mathrm{e}$ of [7]. (Remember: $\Delta_{Z}$ is the intersection of $Z$ with the diagonal in $W \times W$. Meanwhile, $E_{R}, E_{L}$ are the respective intersections of $Z$ with $\gamma \times W$ and $W \times \gamma$; here $\gamma \subset W$ is the pseudo-gradient flow line which starts at $p_{0} \in M_{0}$ and ends at $p_{1} \in M_{1}$.)

\section{a) A first pass at $E_{-}$.}

Recall that the future of a set $U \subset W$ (written fut $(U))$ is the set of points in $W$ which can be reached from $U$ by traveling along a gradient flow line in the direction of increasing $f$.

Introduce

$$
E_{1-}^{\prime} \equiv\left[\left(T_{1} \times \text { fut }\left(T_{2}\right)\right) \cup\left(\text { fut }\left(T_{1}\right) \times T_{2}\right)\right] \cap Z .
$$

Equations (3.6) and (3.20) ensure that $T_{1} \times$ fut $\left(T_{2}\right)$ and fut $\left(T_{1}\right) \times T_{2}$ intersect $Z$ transversally, each as a smooth submanifold with boundary. These assertions are proved with the following fact: Let $U \subset W$ be a submanifold which intersects no pseudo-gradient flow line more than once, and which is nowhere tangent to a pseudo-gradient flow line. Then fut $(U) \subset W$ is a smooth submanifold with boundary, and that boundary is $U$. 
b) $E_{1-}^{\prime}$ as a cycle.

To consider $E_{1-}^{\prime}$ as a cycle, it is necessary to understand first the boundaries of $\left(T_{1} \times\right.$ fut $\left.\left(T_{2}\right)\right) \cap Z$ and (fut $\left.\left(T_{1}\right) \times T_{2}\right) \cap Z$. One finds

$$
\partial\left[\left(T_{1} \times \operatorname{fut}\left(T_{2}\right)\right) \cap Z\right]=\left[\left(T_{1} \times T_{2}\right) \cap Z\right] \cup\left[\left(T_{1} \times \text { fut }\left(T_{2}\right)\right) \cap \partial Z\right],
$$

and, likewise,

$$
\left.\partial\left[\left(\operatorname{fut}\left(T_{1}\right) \times T_{2}\right) \cap Z\right]=\left[\left(T_{1} \times T_{2}\right) \cap Z\right] \cup\left[\left(\operatorname{fut}\left(T_{1}\right) \times T_{2}\right)\right) \cap \partial Z\right] .
$$

(The conditions in (3.6) and (3.20) are used here.)

It follows from (4.3), (4.4) that orientations exist for both (fut $\left.\left(T_{1}\right) \times T_{2}\right) \cap Z$ and $\left(T_{1} \times\right.$ fut $\left.\left(T_{2}\right)\right) \cap Z$ such that $\partial\left[E_{1-}^{\prime}\right]$ has support (as a cycle) in $\left(S^{3} \times S^{3}\right)_{b_{1}} \cup$ $\left(S^{3} \times S^{3}\right)_{b_{2}}$. With the preceding understood, write

$$
E_{1-}^{\prime} \cap \partial Z \equiv S_{b_{1}+} \cup S_{b_{2}-} .
$$

where, $S_{b_{1}}+\subset\left(S^{3} \times S^{3}\right)_{b_{1}}$ while $S_{b_{2}}-\subset\left(S^{3} \times S^{3}\right)_{b_{2}}$. It is left as an exercise to prove that $S_{b_{1}}$ c can be identified as being some number of push-off copies of the right-hand sphere, $\left(S^{3}\right)_{b_{1}}+\subset\left(S^{3} \times S^{3}\right)_{b_{1}}$; while $S_{b_{2}}$ - consists of some number of disjoint, push-off copies of $\left(S^{3}\right)_{b_{2}}-\subset\left(S^{3} \times S^{3}\right)_{b_{2}}$. (See the proof of Lemma 4.1, below.)

The next task is to determine the homology classes of the cycles that $S_{b_{1}+}$ and $S_{b_{2}}$ - define.

Lemma 4.1. The components $\left(T_{1} \times\right.$ fut $\left.\left(T_{2}\right)\right) \cap Z$ and (fut $\left.\left(T_{1}\right) \times T_{2}\right) \cap Z$ of $E_{1-}^{\prime}$ can be oriented so that as a cycle,

$$
\partial\left[E_{1-}^{\prime}\right]=m \cdot\left[S^{3}\right]_{b_{1}+}+m \cdot\left[S^{3}\right]_{b_{2}-} .
$$

Proof. Orient $E_{1-}^{\prime}$ as follows: To begin, orient $T_{1}$ and $T_{2}$ to make their intersection number $\left[T_{1}\right] \cdot\left[T_{2}\right]$ equal to $m$. Let $o_{1,2} \in \Lambda^{2} T\left(T_{1,2}\right)$ denote the respective orientations. Next, orient fut $\left(T_{1}\right)$ and fut $\left(T_{2}\right)$ by using $-v \wedge o_{1,2}$, where $\mathrm{v}$ is the pseudo-gradient for $f$. (Note that $v$ is tangent to $\operatorname{fut}\left(T_{1,2}\right)$ and is inward pointing along $T_{1}$ or $T_{2}$.) Orient $T_{1} \times$ fut $\left(T_{2}\right)$ as $\pi_{L} * o_{1} \wedge \pi_{R} *\left(-v \wedge o_{2}\right)$ and orient fut $\left(T_{1}\right) \times T_{2}$ as $\pi_{L} *\left(v \wedge o_{1}\right) \wedge \pi_{R} * o_{2}$. Notice that the former is oriented using the product orientation, but the latter is oriented in reverse. This insures that the respective orientations which are induced on $T_{1} \times T_{2}$ are, in fact, opposite.

Near $b_{1}, T_{1}$ is identified with $m_{1}$ like oriented, push-off copies of the descending disk $B_{b_{1}}$.. Using the Morse coordinates of (3.2) in [7], this descending disk is given by setting $x_{3}=x_{4}=0$. And, one can assume, without loss of generality, that $o_{1}=\partial_{x_{1}} \wedge \partial_{x_{2}}$. Here, the orientation for $W$ can be assumed to be $o \equiv \partial_{x_{1}} \wedge \partial_{x_{2}} \wedge \partial_{x_{3}} \wedge \partial_{x_{4}}$.

Near $b_{1}$, fut $\left(T_{2}\right)$ is a union of some number of disjoint components. These components can be described as follows: The pseudo-gradient flow isotopes $T_{2}$ 
to where $f \geq 7 / 16$ in $W$. This isotopic image, $\underline{T}_{2}$, intersects $T_{1}$ transversally; in

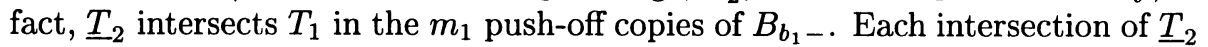
with $B_{b_{1}}$ - defines a component fut $\left(T_{2}\right)$ near $b_{1}$, and likewise each intersection point of $\underline{T}_{2}$ with one of the $m_{1}$ push-off copies of $B_{b_{1}}$ - defines a component of $T_{1} \times$ fut $\left(T_{2}\right)$ near $\left(b_{1}, b_{1}\right)$. Thus, the intersection points of $\underline{T}_{2}$ with the $m_{1}$ push-off copies of $B_{b_{1}}$ - are in 1-1 correspondence with the components of $S_{b_{1}+}$.

Using Morse coordinates of (3.2) in [7] near $b_{1}$, a typical component of fut $\left(T_{2}\right)$ near $b_{1}$ is given by $\left\{x: x_{1}=0, x_{2}>0\right\}$. If this component corresponds to a positive intersection point of $\underline{T}_{2}$ with $T_{1}$, then this component can be assumed oriented by $-\partial_{x_{2}} \wedge \partial_{x_{3}} \wedge \partial_{x_{4}}$; here $\partial_{x_{2}}$ is equal to $v$ where $x_{1}, x_{3}$ and $x_{4}$ all vanish and $x_{2}>0$. Thus, the corresponding component of $T_{1} \times$ fut $\left(T_{2}\right)$ is oriented by

$$
-\partial_{x_{1}} \wedge \partial_{x_{2}} \wedge \partial_{y_{2}} \wedge \partial_{y_{3}} \wedge \partial_{y_{4}}
$$

where

$$
x_{1}=x_{3}=x_{4}=y_{1}=y_{3}=y_{4}=0 \text { and } y_{2}>0 .
$$

Here, the orientation for the intersection of $T_{1} \times$ fut $\left(T_{2}\right)$ with $Z$ is given by contracting (4.7) with $-d y_{2}+d x_{2}$. The resulting orientation is $\partial_{x_{1}} \wedge\left(\partial_{x_{2}}+\partial_{y_{2}}\right) \wedge \partial_{y_{3}} \wedge \partial_{y_{4}}$. The induced boundary orientation is given by contracting this with $-d x_{2}-d y_{2}$; and the result is $\partial_{x_{1}} \wedge \partial_{y_{3}} \wedge \partial_{y_{4}}$.

Meanwhile, $\left(S^{3}\right)_{b_{1}+}=\left\{(x, y): y_{1}=x_{3}=x_{4}=0, x_{2}=r\right\}$. At the point in (4.8), $\left(S^{3}\right)_{b_{1}+}$ is oriented by $\partial_{x_{1}} \wedge \partial_{y_{3}} \wedge \partial_{y_{4}}$ also. Notice that this orientation is the same as that of the boundary of the given component of $T_{1} \times \operatorname{fut}\left(T_{2}\right)$, and this component, by assumption, corresponds to a positive intersection point between $T_{1}$ and $\underline{T}_{2}$.

To summarize the preceding, a component of $S_{b_{1}+}$ is oriented the same as $\left(S^{3}\right)_{b_{1}+}$ if the corresponding intersection point between $T_{1}$ and $\underline{T}_{2}$ is positive; while it is oriented in reverse if the corresponding intersection point between $T_{1}$ and $\underline{T}_{2}$ is negative. This observation justifies the factor of $m$ in the first term on the right side of (4.6) because the algebraic intersection number between $T_{1}$ and $\underline{T}_{2}$ is equal to that between $T_{1}$ and $T_{2}$, which is $m$.

Consider now the analogous calculation near $\left(b_{2}, b_{2}\right)$. Here, the roles of $T_{1}$ and $T_{2}$ are interchanged. The intersection of $T_{1}$ and $T_{2}$ occur along $B_{b_{2}-}^{\prime}$; the $m_{2}$ push-off copies of $B_{b_{2}-}$. Thus, the components of fut $\left(T_{1}\right) \times T_{2}$ near $\left(b_{2}, b_{2}\right)$ are in 1-1 correspondence with the intersection points of $T_{1}$ and $B_{b_{2}-}^{\prime}$ as are the components of $S_{b_{2}-}$.

Use the Morse coordinates of (3.2) in [7] near $b_{2}$. A typical component of $\operatorname{fut}\left(T_{1}\right) \times T_{2}$ near $\left(b_{2}, b_{2}\right)$ is given as

$$
\left\{(x, y): x_{1}=y_{3}=y_{4}=0, x_{2}>0\right\} .
$$

If the component above corresponds to an intersection point of $T_{1}$ with $B_{b_{2}-}^{\prime}$ which has positive intersection number, then the orientation of (4.9) is given by $\partial_{x_{2}} \wedge \partial_{x_{3}} \wedge \partial_{x_{4}} \wedge \partial_{y_{1}} \wedge \partial_{y_{2}}$ at points where

$$
x_{1}=x_{3}=x_{4}=y_{1}=y_{3}=y_{4}=0 \text { and } x_{2}>0 \text {. }
$$


The orientation for the intersection of (4.9) with $Z$ is given by contracting its orientation with $-d y_{2}+d x_{2}$. The resulting orientation at (4.10) is $\partial_{x_{3}} \wedge \partial_{x_{4}} \wedge$ $\partial_{y_{1}} \wedge\left(\partial_{y_{2}}+\partial_{x_{2}}\right)$. The boundary orientation is obtained by contracting again with $-d x_{2}-d y_{2}$; the result is $\partial_{x_{3}} \wedge \partial_{x_{4}} \wedge \partial_{y_{1}}$. Note that this orientation equals the given orientation on $\left(S^{3}\right)_{b_{2}-}$.

The preceding is summarized as follows: A component of $S_{b_{2}-}$ is oriented as $\left(S^{3}\right)_{b_{2}-}$ if the corresponding intersection point of $T_{1}$ and $T_{2}$ is positive; and the component is oriented negatively if the corresponding intersection point is negative. Thus, the factor of $m$ in the second term on the right in (4.6) also follows from the fact that $T_{1} \cdot T_{2}=m$.

\section{c) $E_{1-}$ as a smoothing of $E_{1-}^{\prime}$.}

As defined by (4.2), $E_{1-}^{\prime}$ is the union of a pair of 4-dimensional submanifolds with boundary in $Z$ which meet along a common boundary component which is $\left(T_{1} \times T_{2}\right) \cap Z$. There are no obstructions to smoothing the crease along $\left(T_{1} \times\right.$ $\left.T_{2}\right) \times Z$ to obtain a smoothly embedded, oriented submanifold with boundary, $E_{1-} \subset Z$. The next few subsections will describe some additional properties of $E_{1-}$.

d) $E_{1+}$.

Introduce the switch map

$$
\Theta: W \times W \longrightarrow W \times W
$$

which interchanges the coordinates. This map preserves $Z$. Define $E_{1+}^{\prime} \equiv$ $\Theta\left(E_{1-}^{\prime}\right)$ and $E_{1+} \equiv \Theta\left(E_{1-}\right)$. Thus,

$$
E_{1+}^{\prime}=\left[\left(\operatorname{fut}\left(T_{2}\right) \times T_{1}\right) \cup\left(T_{2} \times \text { fut }\left(T_{1}\right)\right)\right] \cap Z .
$$

\section{e) The intersection with $\Delta_{Z}$.}

Make the standard identification of $\Delta_{W} \subset W \times W$ with $W$ (project on either right of left factor). This identifies $\Delta_{Z}$ with the compliment in $W$ of the union of an open ball about $b_{1}$ and an open ball about $b_{2}$. And this identifies $E_{1-}^{\prime} \cap \Delta_{Z}$ with the intersection of

$$
\left(T_{1} \cap \operatorname{fut}\left(T_{2}\right)\right) \cup\left(\text { fut }\left(T_{1}\right) \cap T_{2}\right)
$$

with the compliment in $W$ of said balls.

To begin the analysis of (4.13), note that fut $\left(T_{1}\right) \cap T_{2}$ is the union of a finite set of half-open arcs which start at the points of $T_{1} \cap T_{2}$ (this is the same as $\left.R_{1} \cap B_{b_{2}-}^{\prime}\right)$. The closure of each of these half-open arcs is an embedded arc whose other endpoint is in $B_{b_{2}-}^{\prime} \cap B_{b_{2}+}$. Remark that there are at least $m$ such arcs.

The intersection of $T_{1}$ with fut $\left(T_{2}\right)$ is the image under the embedding $\varphi$ in (3.16) of $T_{1}^{\prime \prime} \cap$ fut $\left(T_{2}\right)$. The latter is described in Section 3d. 
It follows from the description in Section $3 \mathrm{~d}$ of $T_{1}^{\prime \prime} \cap$ fut $\left(T_{2}\right)$ that the intersection of $E_{1-}$ with $\Delta_{Z}$ is the disjoint union of some number of arcs and some number of circles. The end-points of the arcs lie $\partial E_{1-} \cap \Delta_{Z}$, that is, on $\left(S^{3} \times S^{3}\right)_{b_{1}} \cup\left(S^{3} \times S^{3}\right)_{b_{2}}$. It is important to note that there are at least $\mathrm{m}$ such arcs which join $m$ points of $S_{b_{1}} \cap\left(\Delta_{S^{3}}\right)_{b_{1}}$ with m points of $S_{b_{2}} \cap\left(\Delta_{S^{3}}\right)_{b_{2}}$.

Furthermore, the proof of Lemma 4.1 shows that for at least $m$ of these arcs, the one end point in $S_{b_{1}}+$ and the other in $S_{b_{2}-}$ lie in components which are oriented positively with respect to the given orientations of $\left(S^{3}\right)_{b_{1}}+$ and $\left(S^{3}\right)_{b_{2}-}$, respectively. In fact, there is an arc, $v \subset E_{1-} \cap \Delta_{Z}$, which connects a positively oriented component of $S_{b_{1}+}$ with a positively oriented component of $S_{b_{2}-}$, and which is characterized as follows: Before smoothing $E_{1-}^{\prime}$ to $E_{1-}$, this $v$ was an arc in $E_{1-}^{\prime}$ which intersected $T_{1} \cap$ fut $\left(T_{2}\right)$ as $\varphi\left(v^{0}\right) \cap \Delta_{Z}$, where $v^{0}$ is the half-open arc in (3.14).

\section{f) Intersections with $E_{L, R}$.}

The submanifold $E_{1-}$ can be assumed to have empty intersection with $E_{L, R}$. Indeed, the flow line $\gamma$ between $p_{0} \in M_{0}$ and $p_{1} \in M_{1}$ misses a small ball around $b_{1}$ and $b_{2}$; and a small perturbation of $R_{1}$ and $R_{2}$ will insure that $\gamma$ misses these surfaces also.

\section{g) Normal framings.}

The claim here is that $E_{1-}$ has trivial normal bundle in $Z$, and that there is a trivialization of said normal bundle which restricts to each component of $S_{b_{1}+}$ and $S_{b_{2}}$ as the constant normal framing. (Recall from [7] that the constant framing of $S^{3} \times$ point in $S^{3} \times S^{3}$ is the normal framing which is given by $\pi_{R} * f$, where $\pi_{R}$ maps $S^{3} \times S^{3}$ onto the right factor of $S^{3}$, and $f$ is a normal framing of the point.)

The establishment of this claim requires the following six steps.

Step 1: This first step identifies $E_{1-}$ :

LeMma 4.2. The submanifold $E_{1-}$ is diffeomorphic to the compliment in $T_{1} \times T_{2}$ of a finite number of disjoint, open balls.

Proof. The identification of $E_{1-}$ starts with the identification

$$
\left[\operatorname{fut}\left(T_{1}\right) \times T_{2}\right] \cap Z \approx\left(R_{1} \times B_{b_{2}-}^{\prime}\right)-U
$$

where $U$ is a finite set of disjoint, open balls. Meanwhile,

$$
\begin{aligned}
& {\left[T_{1} \times \operatorname{fut}\left(T_{2}\right)\right] \cap Z} \\
& \quad \approx\left[\left(R_{1} \times R_{2}\right) \cup\left(B_{b_{1}-}^{\prime} \times R_{2}\right) \cup\left(B_{b_{1}-}^{\prime} \times B_{b_{2}-}^{\prime}\right)\right]-U^{\prime} .
\end{aligned}
$$

Here, $R_{1} \times R_{2}$ and $B_{b_{1}-}^{\prime} \times R_{2}$ are attached along their common boundary component, $\partial B_{b_{1}-}^{\prime} \times R_{1}$. Meanwhile, $\left(B_{b_{1}-}^{\prime} \times R_{2}\right) \cup\left(B_{b_{1}-}^{\prime} \times B_{b_{2}-}^{\prime}\right)$ are attached along their common boundary component, $B_{b_{1}-}^{\prime} \times \partial B_{b_{2}-}^{\prime}$. Finally, $U^{\prime} \subset \operatorname{int}\left(B_{b_{1}-}^{\prime} \times R_{2}\right)$ is a finite, disjoint collection of open balls. 
Remember that $\left(\right.$ fut $\left.\left(T_{1}\right) \times T_{2}\right) \cap Z$ and $\left(T_{1} \times\right.$ fut $\left.\left(T_{2}\right)\right) \cap Z$ are attached along their common boundary to obtain $E_{1-}$. This common boundary is

$$
\left(T_{1} \times T_{2}\right) \cap Z \approx\left[R_{1} \times\left(S^{1}\right)^{m_{2}}\right] \cup\left[\partial R_{1} \cup B_{b_{2}-}^{\prime}\right],
$$

where $\left(S^{1}\right)^{m_{2}} \approx\left(B_{b_{2}-}^{\prime} \cap \underline{M}\right)$. With (4.16) understood, one can see (4.14) and (4.15) as a decomposition of $T_{1} \times T_{2}$ less some number of open balls by writing $T_{1} \approx B_{b_{1}-}^{\prime} \cup R_{1}$ and $T_{2} \approx B_{b_{2}-}^{\prime} \cup R_{2}$.

Step 2: The normal bundle to $E_{1-}$ in $Z$ is an oriented three-plane bundle, and since $E_{1-}$ is not closed, this 3-plane bundle is classified by its 2nd StieffelWhitney class, $w_{2}$. This class is zero for the following reasons: First, $w_{2}(T W)$ $=0$ since $W$ is assumed to be a spin manifold. Thus, $w_{2}(T(W \times W))=0$. Second, remark that $T(W \times W) \mid Z \approx T Z \oplus \underline{\mathbb{R}}$, where $\underline{\mathbb{R}}$ is the trivial, real line bundle. Thus, $w_{2}(T Z)=0$. Restricted to $E_{1-}, T Z \approx T E_{1-} \oplus v E_{1-}$, where $v E_{1-}$ is the normal bundle in question. Now, $T_{1} \times T_{2}$ is a spin manifold, and therefore $w_{2}\left(E_{1-}\right)=0$; so $w_{2}\left(v E_{1-}\right)=0$ as claimed.

Step 3: Having established that $E_{1-}$ has trivial normal bundle in $Z$, it remains yet to establish that this normal bundle has a trivialization which restricts to each component of $\partial E_{1-}$ as the constant normal framing. Here is an outline of the argument:

a) Remember that $E_{1-}$ is the image of an embedding of the compliment in $T_{1} \times T_{2}$ of some number of open balls. With this understood, the proof establishes that this embedding extends as an embedding of $T_{1} \times T_{2}$ into $W \times W$. This extension will be called $\hat{E}_{1-}$.

b) The proof establishes that the normal bundle in $W \times W$ to $\hat{E}_{1-}$ splits as $N \oplus \underline{\mathbb{R}}$, where $N$ is a trivial 3-plane bundle, and where $\underline{\mathbb{R}}$ restricts to $E_{1-} \subset \hat{E}_{1-}$ as the normal bundle to $Z$ in $W \times W$.

c) The proof establishes that $N$ is a trivial 3-plane bundle over $\hat{E}_{1-}$.

d) Thus, $N$ restricts to $E_{1-}$ as $v E_{1-}$; and the restriction of a framing of $N$ to $E_{1-}$ gives a framing of $v E_{1}$ - which is homotopic to the constant framing over each component of $\partial E_{1-}$.

Step 4: To esteblish Step 3a, above, remark that a component, $C$ of $\partial E_{1-}$ on $\left(S^{3} \times S^{3}\right)_{b_{1}}$ has a neighborhood in $E_{1}$ - which can be assumed to have the following form in coordinates from Lemma 3.6 in [7]:

$$
\left\{(x, y): x_{3}=x_{4}=y_{2}=0 \text { and } x_{1}^{2}+x_{2}^{2}+y_{3}^{2}+y_{4}^{2}=y_{1}^{2}\right\}
$$

where $y_{1} \geq(r / 2)^{1 / 2}$. Here, $C$ is given by (4.17) with $y_{1}=(r / 2)^{1 / 2}$.

Note that $C$ is the intersection with $\left(S^{3} \times S^{3}\right)_{b_{1}}$ of a push-off of the ascending 4-ball from the critical point $\left(b_{1}, b_{1}\right)$ for the function $F$ on $W \times W$ which is given in (3.20) of [7]. Thus $C$ bounds an embedded 4-ball in $W \times W$, for example, the ball $B \subset(W \times W-Z)$ which is given by

$$
\begin{array}{r}
\left\{(x, y): x_{3}=x_{4}=y_{2}=0, y_{1}=(r / 2)_{1 / 2}\right. \\
\text { and } \left.x_{1}^{2}+x_{2}^{2}+y_{3}^{2}+y_{4}^{2} \leq r / 2\right\} .
\end{array}
$$


Each boundary component of $E_{1-}$ has its analogous $B$; and these can be taken to be mutually disjoint, being all push-off copies of a descending 4-ball for $F$ from $\left(b_{1}, b_{1}\right)$ or from $\left(b_{2}, b_{2}\right)$. Glue these 4-balls to $E_{1-}$ along their common boundaries and smooth the corner along $\partial E_{1-}$ to obtain $\hat{E}_{1-}$, an embedding of $T_{1} \times T_{2}$ into $W \times W$ which extends $E_{1-}$.

Step 5: To establish Step 3b, note that the normal bundle to $\hat{E}_{1-}$ in $W \times W$ splits as $v E_{1-} \oplus \underline{\mathbb{R}}$ where $\underline{\mathbb{R}}$ is spanned by a section of $T(W \times W)$ along $E_{1-}$ which has positive pairing with $d F$. With this understood, consider the vector field $-\partial / \partial y_{1}$ in the coordinates of (4.17), (4.18). This vector field is nowhere tangent to $\hat{E}_{1-}$ and restricts to a neighborhood of $B$ in $\hat{E}_{1-}$ to have positive pairing with the 1 -form $d F$. Thus, $-\partial / \partial y_{1}$ extends the preceding splitting of the normal bundle of $E_{1-}$ in $W \times W$ to a splitting of the normal bundle of $\hat{E}_{1-}$ in $W \times W$ as $N \oplus \underline{\mathbb{R}}$, where $N=v E_{1-}$ over $E_{1-}$.

Step 6: The fact that $v E_{1-}$ is trivial implies that $w_{2}(N)=0$. Thus, $N$ is the trivial bundle if $N$ 's first Pontrjagin class vanishes. This class is computed as follows: Since $p_{1}\left(T\left(T_{1} \times T_{2}\right)\right)=0$, it follows that $p_{1}(N)$ is the same as $p_{1}(T(W \times W)) \mid \hat{E}_{1-}$. Thus, $N$ is trivial if $p_{1}(T(W \times W))$ is trivial as a rational class. The latter is trivial because $\left.p_{1}(T(W \times W)) \approx \pi_{L} * p_{1}(T W)+\pi_{R} * p_{1}(T W)\right)$, and both these classes vanish because $W$ is has non-trivial boundary.

\section{h) A fiducial homotopy class of normal framing.}

The previous subsection establishes that there are homotopy classes of normal framings for $E_{1-}$ in $Z$ which restrict to each component of $\partial E_{1-}$ as the class of the constant normal framing. The purpose of this subsection is to describe a subset of such classes which behave nicely when restricted to a specific set of generators for $H_{1}\left(E_{1-}\right)$.

To make this all precise, it proves useful to first digress to describe a set of generators of $H_{1}\left(E_{1-}\right)$. To begin the digression, take the generators $\left\{\eta_{1 \alpha}\right\}$ for $H_{1}\left(T_{1}^{\prime \prime}\right)$ and $\left\{\eta_{2 \beta}\right\}$ for $H_{1}\left(T_{2}\right)$ as described in (3.15). Choose a point $x_{1} \in R_{1}$ and a point $x_{2} \in R_{2}$. Then, generators for $H_{1}\left(E_{1-}\right)$ are given by

1) $\left\{s_{1 \alpha} \equiv\left(\varphi\left(\eta_{1 \alpha}\right) \times\right.\right.$ fut $\left.\left.\left(x_{2}\right)\right) \cap Z\right\}$,

2) $\left\{s_{2 \beta} \equiv\left(x_{1} \times\right.\right.$ fut $\left.\left.\left(\eta_{2 \beta}\right)\right) \cap Z\right\}$.

Fix generators $\left\{s_{1 \alpha}, s_{2 \beta}\right\}$ as above. End the digression.

Ideally, a normal frame for $E_{1}$ - should restrict to these circles as a product normal frame, $e=\left(e_{1}, e_{2}, e_{3}\right)$, for $R_{1} \times$ fut $\left(R_{2}\right)$ in $W \times W$ with the following properties:

1) $e_{1}$ is normal to $\varphi\left(M_{3 / 8}\right)$ in $W$ and $\left\langle d f, e_{1}\right\rangle<0$.

2) $e_{2}$ is normal to $R_{1}$ in $\varphi\left(M_{3 / 8}\right)$.

3) $e_{3}$ is normal to fut $\left(R_{2}\right)$ in $W$ and $\left\langle d f, e_{3}\right\rangle=0$.

LEMMA 4.3. Given generators $\left\{s_{1 \alpha}, s_{2 \beta}\right\}$ for $H_{1}\left(E_{1-}\right)$ as described in (4.19), there is a normal frame for $E_{1-}$ in $Z$ whose restriction to each component of 
$\partial E_{1-}$ is a constant normal frame, and whose restriction to each $s \in\left\{s_{1 \alpha}, s_{2 \beta}\right\}$ is described by (4.20).

Remark: A normal frame for $E_{1-}$ which is described by Lemma 4.3 will be called a fiducial normal frame.

Proof. The restriction of a given normal frame $\xi$ of $E_{1-}$ to $s \in\left\{s_{1 \alpha}, s_{2 \beta}\right\}$ can be written as $g \cdot e$, where $g: s \longrightarrow S O(3)$. If $g$ is null-homotopic, then, and only then can $\xi$ be homotoped to a frame whose restriction to $s$ is equal to $e$. With the preceding understood, note that a map $g: S^{1} \longrightarrow S O(3)$ is classified by the class in $H^{1}\left(S^{1} ; \mathbb{Z} / 2\right)$ of the pull-back of the generator, $\sigma$, of the module $H^{1}(S O(3) ; \mathbb{Z} / 2)$. Therefore, a normal frame $\xi$ for $E_{1-}$ (which is homotopic to a constant frame on each component of $\left.\partial E_{1-}\right)$ defines an element $\lambda(\xi) \in$ $\left(\oplus_{\alpha} H^{1}\left(s_{1 \alpha} ; \mathbb{Z} / 2\right)\right) \oplus\left(\oplus_{\beta} H^{1}\left(s_{2 \beta} ; \mathbb{Z} / 2\right)\right)$ which is the obstruction to deforming $\xi$ to a fiducial frame. By the way, note that when $h: E_{1-} \longrightarrow S O(3)$, then $\lambda(h \cdot \xi)=\lambda(\xi)+i^{*} h^{*} \sigma$, where $i$ is the inclusion map of $\left(\cup_{\alpha} s_{1 \alpha}\right) \cup\left(\cup_{\beta} s_{2 \beta}\right)$ into $E_{1-}$.

To prove the lemma, take a normal frame $\xi$ for $E_{1-}$ and define a map $q$ : $\left(\cup_{\alpha} s_{1 \alpha}\right) \cup\left(\cup_{\beta} s_{2 \beta}\right) \longrightarrow S^{1}$ as follows: If $s \in\left\{s_{1 \alpha}, s_{2 \beta}\right\}$ and $\lambda(\xi)$ has trivial summand in $H^{1}(s ; \mathbb{Z} / 2)$, then make $q \mid s$ the constant map. Otherwise, make $q \mid s$ a diffeomorphism to $S^{1}$ (a degree one map.) Because $\left\{s_{1 \alpha}, s_{2 \beta}\right\}$ generate $H_{1}\left(E_{1-}\right)$, this map $q$ extends as a map $q: E_{1-} \longrightarrow S^{1}$ which is trivial near $\partial E_{1-}$. Let $j: S^{1} \longrightarrow S O(3)$ generate $H_{1}(S O(3))$ and set $h \equiv j \circ \underline{q}$. Then $\lambda(h \circ \xi)=0$ because of the equalities $i^{*} h^{*} \sigma=(j \circ q)^{*} \sigma=\lambda(\xi)$.

i) $H^{2}\left(E_{1-}\right)$ and $H_{2}\left(E_{1-}\right)$.

Lemma 4.2 implies that

$$
H^{2}\left(E_{1-} ; \mathbb{R}\right) \approx H^{2}\left(T_{1}\right) \oplus H^{2}\left(T_{1}\right) \oplus\left(H^{1}\left(T_{1}\right) \otimes H^{1}\left(T_{2}\right)\right) .
$$

Of course, $H_{2}\left(E_{1-} ; \mathbb{R}\right)$ is isomorphic to $(4.21)$, but the proof of the results in the introductory section requires a set of generators for $H_{2}\left(E_{1-} ; \mathbb{R}\right)$.

To give such generators, it is necessary to first choose orientations for $T_{1}$ and $T_{2}$ so that their intersection number equals $m$. Choose a point $p_{1} \in B_{b_{1}-}^{\prime}$ with $f\left(p_{1}\right)<f \mid T_{2}$. Also, choose a point $p_{2} \in R_{2}$ which is on a gradient flow line which ends on $M_{1}$.

With these choices understood, then

$$
\begin{array}{ll}
\text { 1) } & T_{1-} \equiv\left(T_{1} \times \text { fut }\left(p_{2}\right)\right) \cap Z \\
\text { 2) } & T_{2-} \equiv p_{1} \times\left(\operatorname{fut}\left(T_{2}\right) \cap f^{-1}\left(p_{1}\right)\right)
\end{array}
$$

are embedded submanifolds of $E_{1-}$ each of whose fundamental class is a generator of $H_{2}\left(E_{1-}\right)$.

To obtain the remaining generators, it is necessary to first choose embedded circles, $\left\{\eta_{1 \alpha}\right\} \subset R_{1}^{\prime \prime}$ and $\left\{\eta_{2 \beta}\right\} \subset R_{2}$ which generate the respective first homology of $T_{1}^{\prime \prime}$ and $T_{2}$. Equation (3.15) introduces an integer $n_{1} \geq 1$ and then, for each $\beta$, a set $\left\{\rho_{\beta_{i}}^{\prime}\right\}_{i=1}^{n_{1}}$ of $n_{1}$ like oriented, push-off copies (in $R_{2}$ ) of $\eta_{2 \beta}$. Let $\eta_{2 \beta}^{\prime} \equiv \cup_{i} \rho_{\beta_{i}}^{\prime}$. Orient this submanifold of $M_{1 / 4}$ by taking the given orientation of 
each $\rho_{\beta_{i}}^{\prime}$. For future applications, it should be assumed, as in Section 3e, that $\left\{\eta_{1 \alpha}\right\}$ is disjoint from fut $\left(\left\{\eta_{2 \beta}^{\prime}\right\}\right)$.

For each $\alpha$, fix a set, $\eta_{1 \alpha}^{\prime} \subset R_{1}^{\prime \prime}$, of $n_{1}$, like oriented, push-off copies of $\eta_{1 \alpha}$. Do not make a big push off: The push-off isotopy must not intersect fut $\left(\left\{\eta_{2 \beta}^{\prime}\right\}\right)$ nor should (3.17.5) fail with $\left\{\eta_{1 \alpha}^{\prime}\right\}$ replacing $\left\{\eta_{1 \alpha}\right\}$.

The remaining generators of $H_{2}\left(E_{1-}\right)$ can be taken to be the fundamental classes of

$$
T_{\alpha \beta-} \equiv\left(\varphi\left(\eta_{1 \alpha}^{\prime}\right) \times \operatorname{fut}\left(\eta_{2 \beta}^{\prime}\right)\right) \cap Z
$$

j) Pushing off $H_{2}\left(E_{1-}\right)$.

The second homology of $E_{L}$ with real coefficients is generated by

$$
\left[T_{1 L}\right] \equiv\left[\left(T_{1} \times \gamma\right) \cap Z\right] \text { and }\left[T_{2 L}\right] \equiv\left[\left(T_{2} \times \gamma\right) \cap Z\right]
$$

The second homology of $E_{R}$ with real coefficients is generated by the corresponding $\left[T_{1 R}\right] \equiv \Theta_{*}\left[T_{1 L}\right]$ and $\left[T_{2 R}\right]=\Theta_{*}\left[T_{2 L}\right]$. The inclusion map from $E_{L} \cup E_{R}$ into $Z$ identifies these four classes as generators of $H_{2}(Z)$. (Use real coefficients here and through out this subsection.)

The inclusion map of $E_{1-} \cup E_{R} \cup E_{L}$ into $Z$ induces a homomorphism

$$
\iota: H_{2}\left(E_{1-} \cup E_{L} \cup E_{R}\right) \longrightarrow H_{2}(Z)
$$

with the property that

$$
\begin{array}{ll}
\text { 1) } & \iota \cdot\left(\left[T_{1-}\right]-\left[T_{1 L}\right]\right)=0 \\
\text { 2) } & \iota \cdot\left(\left[T_{2-}\right]-\left[T_{2 R}\right]\right)=0 \\
\text { 3) } & \iota \cdot\left[T_{\alpha, \beta-}\right]=0
\end{array}
$$

As discussed in Section $4 \mathrm{~h}$, the submanifold $E_{1}$ - has a trivial normal bundle in $Z$ with a fiducial homotopy class of framing which restricts to each component of $\partial E_{1-}$ as the class of the constant normal framing. Choose a framing from such a homotopy class and use one of the frame vectors to push each of the submanifolds $T_{1-}, T_{2-}$, and $\left\{T_{\alpha, \beta-}\right\}$ into $Z-E_{1-}$.

If $p_{2}$ is chosen so that fut $\left(p_{2}\right)$ is disjoint from $T_{1}$, then the submanifold $T_{1-}$ is disjoint from $E_{1}+\cup \Delta_{Z}$. If $p_{1}$ is chosen to be disjoint from fut $\left(T_{2}\right)$, then the submanifold $T_{2-}$ is likewise disjoint from $E_{1+} \cup \Delta_{Z}$. As $\left\{\eta_{1 \alpha}\right\}$ and fut $\left(\left\{\eta_{2 \beta}^{\prime}\right\}\right)$ are assumed to be disjoint, $\left\{T_{\alpha, \beta-}\right\}$ is disjoint from $\Delta_{Z}$. And, because of (3.17.5), $\left\{T_{\alpha, \beta_{-}}\right\}$is disjoint from $E_{1+}$. Thus, $T_{1-}, T_{2-}$ and all $\left\{T_{\alpha^{\prime} \beta-}\right\}$ can be pushed off of $E_{1-}$ into $Z-\Sigma_{1}$ where

$$
\Sigma_{1} \equiv \Delta_{Z} \cup E_{L} \cup E_{R} \cup E_{1-} \cup E_{1+}
$$

in an essentially canonical way.

Both $E_{L}$ and $E_{R}$ have a canonical homotopy class of normal bundle framing. The canonical homotopy class of normal framing for $E_{L}$ is the class of the normal framing which is obtained by pulling back via the projection $\pi_{R}$ a normal bundle 
framing for the arc $\gamma$ in $W$. Similarly, the canonical homotopy class of normal framing for $E_{R}$ is obtained by pulling back via the projection $\pi_{L}$ the same normal bundle framing for $\gamma \subset W$. Fix a framing in the canonical homotopy class for $E_{L}$ 's normal bundle and use one of the framing basis vectors to push $T_{1 L}$ and $T_{2 L}$ off of $E_{L}$ into $\Sigma_{1}$. Then, push $T_{1 R}$ and $T_{2 R}$ off of $E_{R}$ into $\Sigma_{1}$ by the analogous method.

These push-offs define a homomorphism

$$
\iota^{\prime}: H_{2}\left(E_{1-} \cup E_{L} \cup E_{R}\right) \longrightarrow H_{2}\left(Z-\Sigma_{1}\right),
$$

and the purpose of this subsection is to prove

Lemma 4.4. The classes $\left(\left[T_{1-}\right]-\left[T_{1 L}\right]\right),\left(\left[T_{2-}\right]-\left[T_{2 R}\right]\right)$ and $\left\{\left[T_{\alpha, \beta-}\right]\right\}$ generate the kernel of $\iota^{\prime}$. Thus, $\operatorname{ker}\left(\iota^{\prime}\right)=\operatorname{ker}(\iota)$.

Proof. The proof considers each of the three kinds of classes in turn.

Case 1: The class $\left[T_{1-}\right]-\left[T_{1 L}\right]$. To begin, remark that there is a natural push-off, $T_{1-}^{\prime}$, of $T_{1-}$ into $\Sigma_{1}$ which is obtained by using (4.22.1) with $p_{2}$ replaced by a point $p_{2}^{\prime} \in M_{1 / 4}-R_{2}$ which is a push-off of $p_{2}$. This sort of push-off can be defined by a normal framing, $\left(e_{1}, e_{2}, e_{3}\right)$, for $E_{1-}$ which has the following property: Along $T_{1-} \subset\left(T_{1} \times\right.$ fut $\left.\left(T_{2}\right)\right) \cap Z$, the frame is the restriction from $T_{1} \times \operatorname{fut}\left(T_{2}\right)$ of a product frame, where

1) $e_{3}$ is normal to $\operatorname{fut}\left(T_{2}\right)$ in $W$ and $\left\langle d f, e_{3}\right\rangle=0$,

2) $\left(e_{1}, e_{2}\right)$ is a normal frame for $T_{1}$ in $W$.

The push-off $T_{1-}^{\prime}$ as described above is then obtained by pushing off $T_{1-}$ along the normal vector $e_{3}$.

Now $T_{1}$ has trivial normal bundle (its self intersection number is zero), so there is a normal frame as in (4.29) for $E_{1-}$ along $T_{1-}$. Furthermore,

Lemma 4.5. There is a fiducial normal frame from Section 4 h whose restriction to $T_{1-}$ is described by (4.29).

This lemma is proved below; accept it for the time being to continue with the proof of Lemma 4.4 for $\left[T_{1-}\right]-\left[T_{1 L}\right]$.

An acceptable push-off of $T_{1 L}$ is defined as follows: Take a point $p_{0}^{\prime} \epsilon M_{1 / 4}$ which is near too, but not equal to $\gamma \cap M_{1 / 4}$. A push-off of $T_{1 L}$ into $Z-\Sigma_{1}$ is $\left(T_{1} \times \operatorname{fut}\left(p_{0}^{\prime}\right)\right) \cap Z$.

Since $R_{2}$ and $R_{1}$ both are connected, and both have non-trivial boundaries, one can find a path $\mu$ in $M_{1 / 4}$ with one endpoint $p_{2}^{\prime}$ and the other $p_{0}^{\prime}$ and whose future is disjoint from $T_{1}, T_{2}$ and $\gamma$. With this understood, then $\left(T_{1} \times\right.$ fut $\left.(\mu)\right) \cap Z$ is an isotopy in $Z-\Sigma_{1}$ between the push-offs of $T_{1-}$ and $T_{1 L}$.

Proof. Let $\xi$ denote a normal frame from Section $4 \mathrm{~h}$. There is a map $g: T_{1-} \longrightarrow S O(3)$ such that $g \cdot\left(\xi \mid T_{1-}\right)$ is described by (4.29). With this understood, the lemma follows if such a map $g$ can be found which is null homotopic. Now, a map $g: T_{1-} \longrightarrow S O(3)$ is null homotopic if and only if the map lifts to a 
map into $S^{3}$. The obstruction to such a lift is an element $\theta(g) \in H^{1}\left(T_{1-} ; \mathbb{Z} / 2\right)$ which is the pull-back by $g$ of the generator of $H^{1}(S O(3) ; \mathbb{Z} / 2)$. Note that $\theta\left(g_{1} \cdot g_{2}\right)=\theta\left(g_{1}\right)+\theta\left(g_{2}\right)$. Store this information.

Consider now the homotopy classes of normal frames which have the form of (4.29). Given that $e_{3}$ is constrained to lie on a fixed side of $R_{2}$, these are in 1-1 correspondence with the homotopy classes of normal frames of $T_{1}$. The latter set is isomorphic (though not canonically) to the set of homotopy classes of maps from $T_{1-}$ to $S O(2) \approx S^{1}$.

Meanwhile, a map $h: T_{1-} \longrightarrow S^{1}$ is distinguished up to homotopy by an invariant $\theta_{1}(h) \in H^{1}\left(T_{1-} ; \mathbb{Z}\right)$ which is the class of the pull-back by $\mathrm{h}$ of the generator of $H^{1}\left(S^{1}\right)$. Furthermore, let $j: S O(2) \longrightarrow S O(3)$ denote the usual inclusion. Then $j \circ h: T_{1-} \longrightarrow S O(3)$ and one has $\theta(j \circ h)=\theta_{1}(h) \bmod (2)$.

The lemma now follows from this last comment because $H^{1}\left(T_{1-} ; \mathbb{Z} / 2\right) \approx$ $H^{1}\left(T_{1-;} ; \mathbb{Z}\right) \otimes \mathbb{Z} / 2$.

Case 2: The class $\left[T_{2-}\right]-\left[T_{2 R}\right]$. The argument for $\left[T_{2-}\right]-\left[T_{2 R}\right]$ is essentially the same as the preceding one and will not be given.

Case 3: The classes $\left\{\left[T_{\alpha, \beta}\right]\right\}$. The first step is to consider the trivialization of the normal bundle of $E_{1-}$ along a given $T_{\alpha, \beta}$.

Lemma 4.6. There are fiducial normal frames for $E_{1-}$ (as defined in Section 4h) that restrict to $T_{\alpha, \beta}$ as the restriction of a normal frame $e=\left(e_{1}, e_{2}, e_{3}\right)$ which is a product normal frame for the submanifold $R_{1} \times$ fut $\left(R_{2}\right) \subset W \times W$ as described by (4.20).

This lemma is proved below; accept it momentarily to continue the proof of Lemma 4.5.

Describe the push-off of $T_{\alpha, \beta-}$ into $Z-\Sigma_{1}$ as follows: Let $M_{3 / 8} \equiv f^{-1}(3 / 8)$. Push $\eta_{1 \alpha}^{\prime}$ into $M_{3 / 8}$ along the pseudo-gradient flow. Use $\underline{\eta}_{1 \alpha}^{\prime}$ to denote the resulting set of circles. Then

$$
\underline{T}_{\alpha, \beta-} \equiv \underline{\eta}_{1 \alpha}^{\prime} \times\left(\operatorname{fut}\left(\eta_{2 \beta}^{\prime}\right) \cap M_{3 / 8}\right)
$$

is a acceptable push-off of $T_{\alpha, \beta-}$ into $Z-\Sigma_{1}$.

Now, remark that $\eta_{1 \alpha}^{\prime}$ bounds a smooth surface $S_{\alpha} \subset \varphi\left(M_{3 / 8}\right)$, and this means that $\underline{\eta}_{\alpha}^{\prime}$ bounds in $M_{3 / 8}$, the bounding surface, $S_{\alpha}$, is obtained by flowing $S_{\alpha}$ along the pseudo-gradient flow lines into $M_{3 / 8}$. With the preceding understood,

$$
\underline{S}_{\alpha} \times\left(\operatorname{fut}\left(\eta_{2 \beta}^{\prime}\right) \cap M_{3 / 8}\right)
$$

bounds $\underline{T}_{\alpha, \beta-}$ in $Z$.

Note that (4.31) is disjoint from $E_{1-}$ and from $E_{1+}$. It is also disjoint from $E_{L}$, and it is disjoint from $E_{R}$ if $S_{\alpha}$ is chosen to miss the point of intersection of $\gamma$ with $\varphi\left(M_{3 / 8}\right)$.

The intersection of (4.31) with $\Delta_{Z}$ is

$$
\left(S_{\alpha} \cap \operatorname{fut}\left(\eta_{2 \beta}^{\prime}\right)\right) \subset \Delta_{Z} .
$$


This may be non-empty. However, by assumption, the linking matrix of Section 3e has all entries zero, which means that the intersection points in (4.32) can be paired so that the local intersection numbers $( \pm 1)$ of the points in each pair cancel. The cancelling of these local signs in pairs implies that an ambient surgery in $Z$ of the interior of (4.32) (remove $\left(S^{0} \times B^{3}\right)$ which intersect $\Delta_{Z}$ and replace with $\left(B^{1} \times S^{2}\right)$ 's which do not) will result in a submanifold with boundary in $Z$ which is completely disjoint from $\Sigma_{1}$ and which bounds $\underline{T}_{\alpha, \beta-}$.

Proof. Upon restriction to $T_{\alpha, \beta}$, a fiducial normal frame $\xi$ for $E_{1-}$ (as described in Section $4 \mathrm{~h}$ ) has the form $g \cdot e$ for some $g: T_{\alpha, \beta} \longrightarrow S O(3)$. If $\mathrm{g}$ is null-homotopic, then $\xi$ can be homotoped in a neighborhood of $T_{\alpha, \beta}$ to restrict to $T_{\alpha, \beta}$ as the restriction of $e$.

With the preceding understood, remark that a map $g$ as above is null homotopic if and only if $g$ lifts to a map into $S^{3}$. The obstruction to finding such a lift is $g^{*} \sigma \in H^{1}\left(T_{\alpha, \beta} ; \mathbb{Z} / 2\right)$, where $\sigma$ generates $H^{1}(S O(3) ; \mathbb{Z} / 2)$. Now, $T_{\alpha, \beta}$ is the disjoint union of push-off copies of an embedded torus, $\left(\varphi\left(\eta_{1 \alpha}\right) \times \operatorname{fut}\left(\eta_{2 \beta}\right)\right) \cap Z$. The first homology of this embedded torus is generated by the circles $\left(\varphi\left(\eta_{1 \alpha}\right) \times\right.$ fut $\left.\left(x_{2}\right)\right) \cap Z$ and $\left(x_{1} \times\right.$ fut $\left.\left(\eta_{2 \beta}\right)\right) \times Z$; here $x_{1} \in \varphi\left(\eta_{1 \alpha}\right)$ while $x_{2} \in \eta_{2 \beta}$. This fact with Lemma 4.3 insures that $g^{*} \sigma$ is zero.

\section{k) Intersections with $\underline{L}_{ \pm}$.}

Reintroduce the function $F$ on $W \times W$ which assigns $f(y)-f(x)$ to a point $(x, y)$. As remarked in Section 3 of [7], the critical points of $F$ are the points $(p, q)$ where $p$ and $q$ are critical points of $f$. The descending 4-ball from the point $\left(b_{2}, b_{1}\right)$ intersects $Z$ as an embedded 3 -sphere which will be denoted by $S_{(2,1)}$. (In (3.32) of [7], this 3-sphere is denoted by $S_{\left(b_{2}, b_{1}\right)-;}$ but such notation is not necessary here.) Likewise, the ascending 4-ball from $\left(b_{1}, b_{2}\right)$ intersects $Z$ as an embedded 3-sphere which will be denoted by $S_{(1,2)}$ (rather than $S_{\left(b_{1}, b_{2}\right)+}$ as in (3.33) of [7]).

The purpose of this subsection is to prove

LEMMA 4.7. The intersection numbers of $E_{1-}$ and of $E_{1+}$ with $S_{(2,1)}$ add up to zero. The intersection numbers of $E_{1-}$ and of $E_{1+}$ with $S_{(1,2)}$ also add up to zero.

Proof. To consider the case of $S_{(2,1)}$, note that the descending ball from $\left(b_{2}, b_{1}\right)$ is $B_{b_{2}}+\times B_{b_{1}-}$. The intersection numbers of $E_{1-}$ and of $E_{1+}$ with $S_{(2,1)}$ (in $Z$ ) are minus the respective intersection numbers (in $W \times W$ ) of $E_{1-}$ and of $E_{1+}$ with $B_{b_{2}}+\times B_{b_{1}-}$.

Consider first the intersection number of $E_{1-}$ with $B_{b_{2}+} \times B_{b_{1}-}$. There are no intersection points in (fut $\left.\left(T_{1}\right) \times T_{2}\right) \cap Z$ because the intersection between $B_{b_{1}-}$ and $T_{2}$ occurs near $f^{-1}(1 / 4)$, while on fut $\left(T_{1}\right), f \geq 3 / 8$.

As for $\left(T_{1} \times \operatorname{fut}\left(T_{2}\right)\right) \cap Z$, note that $B_{b_{2}}+$ has intersection number $m_{1,2}$ with $T_{1}$; one intersection point is in each of the $\left|m_{1,2}\right|$ copies of $B_{b_{2}-}$ which sit in $B_{b_{1}-}^{\prime}$. Each of these intersection points can be assumed to have a different value of $f$, but all such values occur near $f\left(b_{2}\right)$. Meanwhile, $B_{b_{1}}$ - has intersection 
number $m / m_{1}$ with fut $\left(T_{2}\right) \cap f^{-1}\left(f\left(b_{2}\right)\right)$. This number is computed using the following facts:

1) The intersection number of $T_{1}$ with fut $\left(T_{2}\right) \cap f^{-1}\left(f\left(b_{2}\right)\right.$ is the same as that of $T_{1}$ with $T_{2}$.

2) $T_{1}$ intersects fut $\left(T_{2}\right) \cap f^{-1}\left(f\left(b_{2}\right)\right)$ only in the push-off copies of $B_{b_{1}-}$ in $\varphi\left(B_{b_{1}-}^{\prime \prime}\right)$.

Thus, $B_{b_{2}+} \times B_{b_{1}-}$ has intersection number $m \cdot m_{1,2} / m_{1}$ with $E_{1-}$ (so $S_{(2,1)}$ has intersection number equal to $-m \cdot m_{1,2} / m_{1}$ with $\left.E_{1-}\right)$.

Now turn to the intersection number of $B_{b_{2}}+\times B_{b_{1}}$ - with $E_{1+}$. Here, there are no intersections in (fut $\left.\left(T_{2}\right) \times T_{1}\right) \cap Z$ because $B_{b_{2}}+$ intersects fut $\left(T_{2}\right)$ where $f>7 / 16$, while $B_{b_{1}}$ - intersects $T_{1}$ where $f$ is approximately $3 / 8$.

On the other hand, $B_{b_{2}}$ has intersection number $m_{2}$ with $T_{2}$, once in each copy of $B_{b_{2}-}$ that makes up $B_{b_{2}-}^{\prime}$. Each such intersection takes place near $b_{2}$. Meanwhile, the intersection number between $B_{b_{1}}$ and fut $\left(T_{1}\right) \cap^{-1}\left(b_{2}\right)$ is equal to $-m \cdot m_{1,2} /\left(m_{1} \cdot m_{2}\right)$. This number is computed using the following facts:

1) $T_{1}$ has zero intersection number with itself.

2) A push-off copy of $T_{1}$ can be constructed which intersects $T_{1}$ as a push-off of $B_{b_{1}-}^{\prime}$ intersects fut $\left(R_{1}\right) \cap f^{-1}(7 / 16)$.

3) $T_{2}$ has $m$ intersections with $T_{1}$, one in each of the push-off copies of $B_{b_{2}-}$ that comprise $B_{b_{2}-}^{\prime}$.

Thus, $B_{b_{2}} \times B_{b_{1}}$ has intersection number $-m \cdot m_{1,2} / m_{1}$ with $E_{1+}$ (so $S_{(2,1)}$ has intersection number $m \cdot m_{1,2} / m_{1}$ with $\left.E_{1+}\right)$.

The case for $S_{(1,2)}$ follows from the preceding computation because $S_{(1,2)}=$ $\Theta\left(S_{(2,1)}\right)$ while $\Theta$ interchanges $E_{1-}$ with $E_{1+}$.

5 The Construction of $E_{ \pm}$. The previous section began the construction of $\Sigma_{Z}$ in the case where $W$ is a cobordism of simple type as described in Definition 2.2. (See (4.1).) This section will finish the construction of $\Sigma_{Z}$ for such a cobordism. Indeed, (4.1) is missing only definitions of $E_{ \pm}$; and this section will construct $E_{ \pm}$from $E_{1 \pm}$ via ambient surgery in $Z$. The surgical techniques here are those from Sections 7-10 of [7].

\section{a) Constructing $E_{2-}:$ Push-offs and tubings.}

Begin with $E_{1-}$ of the preceding section. Using the fiducial normal framing at the end of Section $4 \mathrm{~h}$, make $2 \mathrm{~m}$ disjoint, push-off copies of $E_{1-}$ in addition to the original. Orient the first $\mathrm{m}$ copies as the original, and orient the last $\mathrm{m}$ copies in reverse. Use $E_{2-}^{\prime}$ to denote the resulting disjoint union.

The boundary of $E_{1-}$ is described in (4.5) and (4.6). That is, it is a union of 3-spheres which are push-off copies of $\left(S^{3}\right)_{b_{1}}+$ or of $\left(S^{3}\right)_{b_{2}-}$ in $\left(S^{3} \times S^{3}\right)_{b_{1}}$ or in $\left(S^{3} \times S^{3}\right)_{b_{2}}$, respectively. As described at the end of Section $4 \mathrm{e}$, there is an 
arc component $v$ of $E_{1-} \cap \Delta_{Z}$ that connects a positively oriented component $S_{1} \subset \partial E_{1-} \cap\left(S^{3} \times S^{3}\right)_{b_{1}}$ - with a positively oriented component $S_{2} \subset \partial E_{1-} \cap$ $\left(S^{3} \times S^{3}\right)_{b_{2}}$.

Each of the first $m-1$ push-off copies of $E_{1}$ - contains a push-off copy of $S_{1}$. Let $\left\{S_{1 \alpha}\right\}_{\alpha=0}^{m-1}$ denote this set of push-offs. Here, $S_{10}$ is the original $S_{1}$ in the original copy of $E_{1-}$. Use $\left\{S_{2 \alpha}\right\}_{\alpha=0}^{m-1}$ to denote the corresponding copies of $S_{2}$ (with $S_{20}$ denoting the original), and let $\left\{v_{\alpha}\right\}_{\alpha=0}^{m-1}$ denote the corresponding copies of $v$.

Note that the components of $\left(\partial E_{2-}^{\prime} \cap\left(S^{3} \times S^{3}\right)_{b_{1}}\right)-\left\{S_{1 \alpha}\right\}$ can be paired up so that each pair contains one positively oriented sphere and one negatively oriented sphere. The spheres in each pair should be tubed to each other as described in Section 7b of [7] (see (7.3) in [7]). Note: As $m \geq 1$, there is at least one pair to tube here.

Likewise, the components of $\left(\partial E_{2-}^{\prime} \cap\left(S^{3} \times S^{3}\right)_{b_{2}}\right)-\left\{S_{2 \alpha}\right\}$ can be paired so that each pair contains one positively oriented sphere and one negatively oriented sphere. The spheres in each pair should be tubed to each other as described in the same Section $7 \mathrm{~b}$ of [7]. There is at least one pair to tube here too.

Use $E_{2}$ to describe the submanifold (with boundary) of $Z$ that results. By construction,

$$
\partial E_{2-}=\left\{S_{1 \alpha}\right\}_{\alpha=0}^{m-1} \cup\left\{S_{2 \alpha}\right\}_{\alpha=0}^{m-1} .
$$

Note as well (see Section 7e of [7]) that after a small perturbation, the intersection of $E_{2-}$ with $\Delta_{Z}$ will be transversal, and given by

$$
E_{2-} \cap \Delta_{Z}=\left\{v_{\alpha}\right\}_{\alpha=0}^{m-1} \cup C
$$

where $C \subset \operatorname{int}\left(\Delta_{Z}\right)$ is a disjoint union of embedded circles.

One can argue as in Section $7 \mathrm{f}$ of [7] that $E_{2-}$ has trivial normal bundle in $Z$ with a framing which restricts to each component of $\partial E_{2-}$ as the constant normal framing.

Meyer-Vietoris (as used in the proof of Assertion 6 of Proposition 7.4 in [7]) shows that $H^{2}\left(E_{2-}\right) \approx H^{2}\left(E_{2-}^{\prime}\right)$.

Define $E_{2+} \equiv \Theta\left(E_{2-}\right)$ and define $\Sigma_{2}$ as in (4.27) with $E_{2 \pm}$ instead of $E_{1 \pm}$. Define the homorphism $\iota: H_{2}\left(E_{2-} \cup E_{L} \cup E_{R}\right) \rightarrow H_{2}(Z)$ from the inclusion into $Z$, and define $\iota^{\prime}: H_{2}\left(E_{2} \cup E_{L} \cup E_{R}\right) \longrightarrow H_{2}\left(Z-\Sigma_{2}\right)$ by analogy with (4.28) using the homotopy class of normal frame for $E_{2}$ - which is inherited (as in Section $7 \mathrm{f}$ of [7]) from the canonical homotopy class of normal frame for $E_{1-}$. Then

$$
\operatorname{ker}(\iota)=\operatorname{ker}\left(\iota^{\prime}\right)
$$

just as in Lemma 4.4. To prove (5.3), note first that (5.3) holds for $E_{2-}^{\prime}$ since $E_{2-}^{\prime}$ is the disjoint union of some nurnber of push-off copies of $E_{1-}$. Next, remark that $E_{2-}=E_{2-}^{\prime}$ except near $\partial Z$. Finally, note that the homologies which prove Lemma 4.4 for $E_{1-}$ are made away from $\partial Z$. 
As a final comment about $E_{2-}$, remark that the tubing can be done in such a way that $E_{2-}$ has empty intersection with $E_{L, R}$; and it can be done so that the tubing avoids the spheres $S_{(2,1)}$ and $S_{(1,2)}$ of Lemma 4.7. In any event, the fundamental class [ $E_{2-}$ ] in $H_{4}(Z, \partial Z)$ will equal [ $\left.E_{1-}\right]$.

\section{b) Constructing $E_{3-}$ : Removing circles.}

The goal here is to take some number $N_{1}$ of like oriented, push-off copies of $E_{2-}$ and do surgery on the circles in its intersection with $\Delta_{Z}$. The goal is to obtain a manifold $E_{3-}$ with the following properties:

Proposition 5.1. There is an oriented submanifold (with boundary) $E_{3}-\subset$ $Z$ and an integer $N \geq 1$ with the following properties:

1) The fundamental class $\left[E_{3-}\right]$ in $H_{4}(Z, \partial Z)$ is equal to $m^{-1} \cdot N \cdot\left[E_{1-}\right]$, and, in particular, obeys

$$
\partial\left[E_{3-}\right]=N \cdot\left[S^{3}\right]_{b_{1}+}+N \cdot\left[S^{3}\right]_{b_{2}-} .
$$

2) The boundary of $E_{3-}$ is a submanifold of $\partial Z$, given by

$$
\partial E_{3-}=\left(\cup_{\alpha=1}^{N} S_{1 \alpha}\right) \cup\left(\cup_{\alpha=1}^{N} S_{2 \alpha}\right),
$$

where each $S_{1 \alpha}$ is a push-off copy of $\left(S^{3} \times\right.$ point $) \subset\left(S^{3} \times S^{3}\right)_{b_{1}}$, while each $S_{2 \alpha}$ is a push-off copy of (point $\left.\times S^{3}\right) \subset\left(S^{3} \times S^{3}\right)_{b_{2}}$.

3) $E_{3}$ - has empty intersection with $M_{0} \times M_{0}$ and with $M_{1} \times M_{1}$.

4) $E_{3-}$ has empty intersection with $E_{L}$ and with $E_{R}$.

5) $E_{3-}$ has transversal intersection with $\Delta_{Z}$ and

$$
E_{3-} \cap \Delta_{Z}=\cup_{\alpha=1}^{N} v_{\alpha}
$$

where $\left\{v_{\alpha}\right\}$ are all push-off copies of an arc. Furthermore, for each $\alpha, v_{\alpha}$ has one end point on $S_{1 \alpha} \cap \Delta_{Z}$ and the other on $S_{2 \alpha} \cap \Delta_{Z}$.

6) $E_{3-}$ has trivial normal bundle in $Z$, and this normal bundle has a fiducial frame $\zeta$ which restricts to each $S_{1 \alpha}$ and each $S_{2 \alpha}$ as the constant normal frame.

7) $E_{3-}$ is obtained from the disjoint union, $E_{2-}^{\prime}$, of some number $N_{1}$ of like oriented, push-off copies of $E_{2-}$ by ambient surgery in $Z$ on embedded circles in $E_{2-}^{\prime} \cap \Delta_{Z}$. This surgery naturally identifies $H^{2}\left(E_{3-} ; \mathbb{Q}\right) \approx$ $\oplus N_{1} H^{2}\left(E_{2-}^{\prime} ; \mathbb{Q}\right)$.

8) Define $E_{3+} \equiv \Theta\left(E_{3-}\right)$ and define $\Sigma_{3}$ as in (4.27) with $E_{3 \pm}$ instead of $E_{1 \pm}$. Define the homorphism $\iota: H_{2}\left(E_{3} \cup E_{L} \cup E_{R}\right) \longrightarrow H_{2}(Z)$ from the inclusion into $Z$, and define $\iota^{\prime}: H_{2}\left(E_{3}-\cup E_{L} \cup E_{R}\right) \longrightarrow H_{2}\left(Z-\Sigma_{3}\right)$ by analogy with (4.28) using the homotopy class of normal frame for $E_{3-}$ from Assertion 6, above. Then $\operatorname{ker}(\iota)=\operatorname{ker}\left(\iota^{\prime}\right)$.

9) The intersection numbers of $E_{3-}$ and $E_{3+}$ with the sphere $S_{(1,2)}$ (of Lemma 4.7) sum to zero; and the same is true for the intersection numbers of $E_{3-}$ and $E_{3+}$ with $S_{(2,1)}$. 
Proof. The submanifold $E_{3}$ - is constructed by mimicking the proof of Proposition 8.1 in [7]. To be brief, the first step is to invoke Propositions 8.3 and 8.7 in [7]. Copy the arguments in Sections 8c, $8 \mathrm{~d}$ and $8 \mathrm{e}$ of [7] to verify that the assumptions of Propositions 8.3 and 8.7 can be met with the following choices of $A, B, X$ and $O$ :

$$
\begin{aligned}
& A \text { is the interior of some number } N_{1} \text { of like oriented, } \\
& \text { push-off copies of } E_{2-} \text {. } \\
& \text { 2) } B=\operatorname{int}\left(\Delta_{Z}\right) \text {. } \\
& \text { 3) } X=\operatorname{int}(Z) \text {. } \\
& \text { 4) } O \text { is the compliment in } \operatorname{int}(Z) \text { of the closure of a regular } \\
& \text { neighborhood of } \partial Z \cup v \cup E_{L} \cup E_{R} \text {. }
\end{aligned}
$$

Here, $v \subset \Delta_{Z} \cap E_{1-}$ is described in Section 5a, above; and it is assumed that $O$ does not contain the $N_{1} \cdot m$ push copies of $v$ which are the arc components of $A \cap \Delta_{Z}$. (Note that there is a basis (i.e., $\left[T_{1}\right]$ and $\left[T_{2}\right]$ ) for $B$ 's second homology in which the $B$ 's intersection form is a $2 \times 2$ matrix with zero's on the diagonal. A symmetric, bilinear form with this property is even.)

In proving Assertion 7, note that the union of the circles in $E_{2-}^{\prime} \cap \Delta_{Z}$ is homologically non-trivial because the construction of $E_{2}$ required at least one pair of tubings near each of $\left(S^{3} \times S^{3}\right)_{b_{1,2}}$.

The proof for Assertion 8 of Proposition 5.1 is as follows: The assertion holds with $E_{3-}^{\prime}$ replacing $E_{3-}$ everywhere since $E_{3-}^{\prime}$ is a union of push-off copies of $E_{2-}$. Meanwhile, the surgery which changes $E_{3-}^{\prime}$ to $E_{3-}$ takes place in a regular neighborhood of $\Delta_{Z}$, and the homologies which prove that $\operatorname{ker}(\iota)=\operatorname{ker}\left(\iota^{\prime}\right)$ can be made with support away from $\Delta_{Z}$. (See the proof of Lemma 4.4.)

Assertion 9 of Proposition 5.1 follows from Assertion 1 and Lemma 4.7.

c) Constructing $E_{4 \pm}$; straightening $E_{3+} \cap E_{3-}$.

The intersection of $E_{3+}$ with $E_{3-}$ can be something of a mess. After small perturbations of $E_{3 \pm}$, this intersection has the form

$$
E_{3+} \cap E_{3-}=\Gamma \cup C
$$

where $\Gamma \subset Z$ is the union of $\Gamma_{1} \equiv \cup_{\alpha=1}^{N} v_{\alpha}$ with some $N-1$ like oriented, push-offs of $\Gamma_{1}$ into $Z-\Delta_{Z}$. These push-offs can be assumed as close to $\Delta_{Z}$ as desired. Meanwhile, $C \subset \operatorname{int}(Z)-\Delta_{Z}$ is a disjoint union of embedded circles.

By the way, (5.5) can be established using (3.17.4).

Argue as in Section 9 in [7] to prove that ambient surgery on a pair, $E_{3-}^{\prime}$, of like oriented push-offs of $E_{3-}$, with ambient surgery on a pair, $E_{3+}^{\prime}$, of like oriented push-offs of $E_{3+}$ will result in submanifolds $E_{4 \pm}$ with the following properties:

Proposition 5.2. There are connected, oriented submanifolds (with boundary) $E_{4-} \subset Z$ and $E_{4+} \subset Z$ and an integer $N \geq 1$ with the following properties:

1) The fundamental classes $\left[E_{4 \pm}\right]$ in $H_{4}(Z, \partial Z)$ equal $m^{-1} \cdot N \cdot\left[E_{1 \pm}\right]$ and furthermore obey

$$
\partial\left[E_{4-}\right]=N \cdot\left[S^{3}\right]_{b_{1}+}+N \cdot\left[S^{3}\right]_{b_{2}-}
$$




$$
\partial\left[E_{4+}\right]=N \cdot\left[S^{3}\right]_{b_{1}-}+N \cdot\left[S^{3}\right]_{b_{2}+} .
$$

2) Let $\Theta: Z \longrightarrow Z$ denote the switch map. Near $\partial Z \cup \Delta_{Z}$,

$$
E_{4+}=\Theta\left(E_{4-}\right) \text {. }
$$

3) The boundary of $E_{4-}$ is a submanifold of $\partial Z$, given by

$$
\partial E_{4-}=\left(\cup_{\alpha=1}^{N} S_{1 \alpha}\right) \cup\left(\cup_{\alpha=1}^{N} S_{2 \alpha}\right),
$$

where each $S_{1 \alpha}$ is a push-off copy of $\left(S^{3} \times\right.$ point $) \subset\left(S^{3} \times S^{3}\right)_{b_{1}}$, while each $S_{2 \alpha}$ is a push-off copy of $\left(\right.$ point $\left.\times S^{3}\right) \subset\left(S^{3} \times S^{3}\right)_{b_{2}}$.

4) $E_{4 \pm}$ have empty intersection with $M_{0} \times M_{0}$ and with $M_{1} \times M_{1}$.

5) $E_{4 \pm}$ have empty intersection with $E_{L}$ and with $E_{R}$.

6) $E_{4 \pm}$ have transversal intersection with $\Delta_{Z}$, and

$$
E_{4-} \cap \Delta_{Z}=E_{4+} \cap \Delta_{Z}=\cup_{\alpha=1}^{N} v_{\alpha}
$$

where $\left\{v_{\alpha}\right\}$ are all push-off copies of an arc. Furthermore, for each $\alpha, v_{\alpha}$ has one end point on $S_{1 \alpha} \cap \Delta_{Z}$ and the other on $S_{2 \alpha} \cap \Delta_{Z}$.

7) $E_{4-}$ has transversal intersections with $E_{4+}$. Furthermore,

$$
E_{4-} \cap E_{4+}=\Gamma
$$

where $\Gamma$ is the union of $\Gamma_{1} \equiv \cup_{\alpha=1} N v_{\alpha}$ and some $N-1$ like oriented, push-off copies of $\Gamma_{1}$ in $Z-\Delta_{Z}$.

8) $E_{4 \pm}$ have trivial normal bundles in $Z$, and these normal bundles have frames $\zeta_{ \pm}$with properties which include: The frames $\zeta_{ \pm}$restrict to each boundary component as the constant frame. Furthermore, where Assertion 2 holds, $\zeta_{+}=\Theta *\left(\zeta_{-}\right)$.

9) $E_{4 \pm}$ are obtained from the union, $E_{3 \pm}^{\prime}$, of one or possibly two like oriented, push-off copies of $E_{3 \pm}$ by ambient surgery in int $\left(Z-\Delta_{Z}\right)$ on the circles in $E_{3-}^{\prime} \cap E_{3+}^{\prime}$. These surgeries naturally identify $H^{2}\left(E_{4 \pm} ; \mathbb{Q}\right) \approx$ $\oplus H^{2}\left(E_{3 \pm}^{\prime} ; \mathbb{Q}\right)$.

10) Define $\Sigma_{4}$ as in (4.27) with $E_{4 \pm}$ instead of $E_{1 \pm}$. Define the homorphism $\iota$ : $H_{2}\left(E_{4-} \cup E_{4+} \cup E_{L} \cup E_{R}\right) \rightarrow H_{2}(Z)$ from the inclusion into $Z$, and define $\iota^{\prime}: H_{2}\left(E_{4-} \cup E_{4+} \cup E_{L} \cup E_{R}\right) \longrightarrow H_{2}\left(Z-\Sigma_{4}\right)$ by analogy with (4.28) using the homotopy class of normal frame for $E_{4 \pm}$ from Assertion 7, above. Then $\operatorname{ker}(\iota)=\operatorname{ker}\left(\iota^{\prime}\right)$.

11) The intersection numbers of $E_{4-}$ and $E_{4+}$ with the sphere $S_{(1,2)}$ (of Lem$m a$ 4.7) sum to zero; and the same is true for the intersection numbers of $E_{4-}$ and $E_{4+}$ with $S_{(2,1)}$.

The fact that $Z$ is path connected implies that $E_{4 \pm}$ can be constructed to be path connected. See Lemma 8.10 in [7] and its proof.

Remark that the last assertion of Proposition 5.2 follows from Assertion 1 and Lemma 4.7. The argument for Assertion 9 proceeds as follows: Since $E_{4 \pm}^{\prime}$ are 
disjoint unions of push-off copies of $E_{3 \pm}$, Assertion 9 holds if $E_{4 \pm}^{\prime}$ everywhere replace $E_{4 \pm}$. Now, $E_{4 \pm}$ is constructed by surgery on $E_{4 \pm}^{\prime}$; and these surgeries can be performed away from the generators of $H_{2}$. Furthermore, the surgeries take place in a regular neighborhood of a surface with boundary or 3-ball in $Z$, and so can be performed away from the homologies which establish Assertion 9 for $E_{4 \pm}^{\prime}$. (See the proof of Lemma 4.4.)

\section{d) The meld construction and $E_{ \pm}$.}

This section constructs $E_{ \pm}$from $E_{4 \pm}$ using the meld operation of Section 10 in [7]. In this regard, note that the behavior of $E_{4-}$ near $\partial Z \cup \Delta_{Z}$ is described by (10.2-5) in [7] modulo notation.

To be precise, there is a regular neighborhood $U \subset Z$ of $\partial Z \cap \Delta_{Z}$ such that $E_{4-} \cap U$ is a set $\left\{Y_{\alpha}\right\}_{\alpha=1}^{N}$ (with $N$ from Proposition 5.2), where $\left\{Y_{\alpha \geq 2}\right\}$ are disjoint, like oriented push-off copies of $Y_{1}$. Meanwhile, $Y_{1}$ is the image of a proper embedding into $U$ of the space in (10.3) of [7]; this being the compliment in the open unit 4 -ball of the open balls $B_{ \pm}$of radius $1 / 8$ and centers $( \pm 1 / 4,0,0,0)$. Note here that the boundary of $B_{+}$is mapped diffeomorphically onto $\left(S^{3}\right)_{b_{1}}+\subset\left(S^{3} \times S^{3}\right)_{b_{1}}$, and the boundary of $B_{-}$is likewise mapped onto $\left(S^{3}\right)_{b_{2}-} \subset\left(S^{3} \times S^{3}\right)_{b_{2}}$. Meanwhile, the arc along the $x$-axis between $( \pm 1 / 8,0,0,0)$ is mapped to the arc $v \subset E_{4-} \cap \Delta_{Z}$.

The $\left\{Y_{\alpha \geq 2}\right\}$ are described by (10.4) and (10.5) in [7].

The melded space, $E_{-}$, is then described by (10.8) in [7]. (See also (10.9) and (10.10) in [7].)

As for $E_{+}$, the neighborhood $U$ can be chosen to be invariant under the switch map (4.11) and such that $E_{4+} \cap U=\Theta\left(E_{4-} \cap U\right)$. With this understood, define $E_{+} \cap(Z-U) \equiv E_{4+} \cap(Z-U)$, and define $E_{+} \cap U \equiv \Theta\left(E_{-} \cap U\right)$.

Note that

$$
\begin{array}{ll}
\text { 1) } & {\left[E_{ \pm}\right]=m^{-1} \cdot N \cdot\left[E_{1 \pm}\right] \text { in } H_{4}(Z, \partial Z ; \mathbb{Z}) .} \\
\text { 2) } \partial\left[E_{-}\right]=N \cdot\left[S^{3}\right]_{b_{1}+}+N \cdot\left[S^{3}\right]_{b_{2}-} \\
\text { and } \partial\left[E_{+}\right]=N \cdot\left[S^{3}\right]_{b_{1}-}+\left[S^{3}\right]_{b_{2}+} \\
\text { 3) } H^{2}\left(E_{ \pm} ; \mathbb{Q}\right) \approx H^{2}\left(E_{4 \pm} ; \mathbb{Q}\right) .
\end{array}
$$

6 Completing the proof The purpose of this last section is to complete the proof of Theorem 1.3 along the lines that were outlined in Section 1c. Thus, suppose that $M_{0}$ and $M_{1}$ are compact, oriented 3-manifolds with the rational homology of $S^{3}$. Assume that $M_{0}$ and $M_{1}$ are spin cobordant by a cobordism whose intersection form is equivalent to a sum of metabolics (see (1.3)). As descibed in (1.4), one can find such a cobordism which factors as $W_{1} \cup W_{2} \cup W_{3}$, where $W_{1}$ and $W_{3}$ have the rational homology of $S^{3}$, and where $W_{2}$ has a good Morse function with only index 2 critical points. As in Proposition 2.1, the cobordism $W_{2}$ can be factored as $\cup_{j=1}^{n} W_{2, j}$, where each $W_{2, j}$ is a cobordism of simple type (Definition 2.2) between a pair, $F_{j-1}$ and $F_{j}$, of rational homology spheres. Here, $F_{0} \equiv M_{0}^{\prime}$ and $F_{n} \equiv M_{1}^{\prime}$.

Define $Z_{2} \equiv \cup_{j} Z_{2, j}$, where each $Z_{2, j} \subset W_{2, j} \times W_{2, j}$ is defined as in Section 2c. The identification of $F_{j} \times F_{j} \subset Z_{2, j}$ with $F_{j} \times F_{j} \subset Z_{2, j+1}$ is left implicit here. Use this $Z_{2}$ in (1.5) 
Fix base points in each $F_{j}$. Then define $\left\{\Sigma_{Z_{N}}\right\}$ as in (4.1). With this understood, set $\Sigma_{Z_{2}} \equiv \cup_{j} \Sigma_{Z_{N}}$. after making the implicit boundary identifications. Use this $\Sigma_{Z_{2}}$ in (1.6).

Step 3 of the outline in Section 1c constructs a closed 2-form $\omega_{Z}$ on the compliment of $\Sigma_{Z}$ which obeys $\omega_{Z} \wedge \omega_{Z} \wedge \omega_{Z}=0$ near $\Sigma_{Z}$. The construction of $\omega_{Z}$ proceeds by first constructing a closed 2 -form $\underline{\omega}_{Z}$ on the compliment of $\Sigma_{Z}$ in a regular neighborhood $N_{Z}$ of $\Sigma_{Z}$ in $Z$. The form $\underline{\omega}_{Z}$ will be built so that it satisfies Condition 1 of Lemma 4.2 in [7]. Also, $\underline{\omega}_{Z} \wedge \underline{\omega}_{Z}=0$.

The form will then be extended over the compliment of $\Sigma_{Z}$ of a neighborhood of $\partial Z \cup \Sigma_{Z}$ so that its pull-back to any boundary component $M \times M-\Sigma_{M}$ is a form which computes $I_{2}(M)$. Here $M \times M$ is any of $M_{0} \times M_{0}, M_{1} \times M_{1}$ or any $\left(S^{3} \times S^{3}\right)_{b_{1 j}},\left(S^{3} \times S^{3}\right)_{b_{2 j}}$.

The next question is whether the form $\underline{\omega}_{Z}$ so constructed can be extended over $Z-\Sigma_{Z}$. The author does not know when such is the case. However, it is shown below that there is a closed 2 -form $\mu$ on $N_{Z}$ which obeys $\mu \wedge \mu=0$, which vanishes near $\partial Z$, and is such that $\omega_{Z} \equiv \underline{\omega}_{Z}-\mu$ extends over $Z-\Sigma_{Z}$ as a closed form. Note that such a form will satisfy the third condition in (1.7).

The form $\mu$ will vanish near $\Sigma_{Z_{1}}$ and near $\Sigma_{Z_{3}}$. Furthermore, $\mu$ will be written as $\mu=\Sigma_{j=1} n \mu_{2, j}$, where $\mu_{2, j}$ has compact support in the interior of $Z_{2, j}$. With this understood, the construction of $\mu_{2, j}$ can be made independently for each factor $Z_{2, j}$ which comprises $Z_{2}$.

\section{a) $\underline{\omega}_{Z}$ near $\Sigma_{Z}$ and $\partial Z$.}

The construction of the closed 2-form $\underline{\omega}_{Z}$ on the compliment of $\Sigma_{Z}$ in a regular neighborhood $N_{Z} \subset Z$ of $\Sigma_{Z} \cup \partial Z$ proceeds by mimicking the constructions in Sections 11a - 11i of [7] which construct $\omega_{Z}$ near $\Sigma_{Z}$ when the cobordism between $M_{0}$ and $M_{1}$ has the rational homology of $S^{3}$. The conditions in Theorem 1.3 that $W$ be spin and that the canonical frame be represented by $c$ in the kernel of the homomorphism $\iota_{W}$ arise here.

The verification of Condition 1 of Lemma 4.2 for $\omega_{Z}$ proceeds as in Section $11 \mathrm{k}$ of [7], and the reader is referred there. (But note Assertion 10 of Proposition 5.2.)

\section{b) The obstruction from cobordisms of simple type.}

At this point, the proof of Theorem 1.3 must diverge from the proof of Theorem 2.9 in [7] because the restriction homomorphism $H^{2}(Z) \longrightarrow H^{2}\left(\Sigma_{Z}\right)$ will not generally be surjective. (Use real coefficients here and throughout this section.) Thus, the second part of Lemma 4.2 in [7] can not be invoked. This failure of surjectivity obstructs the extension of $\underline{\omega}_{Z}$ to $Z-\Sigma_{Z}$.

This extension obstruction will be studied by using the fact that restriction to the $Z_{2, j}$ defines isomorphisms $H^{2}(Z) \approx \oplus_{j} H^{2}\left(Z_{2, j}\right)$ and $H^{2}\left(\Sigma_{Z}\right) \approx$ $\oplus_{j} H^{2}\left(\Sigma_{Z_{2, j}}\right)$. (Meyer-Vietoris proves these assertions.) These direct sum decompositions imply that the obstruction to extending $\omega_{Z}$ over $Z-\Sigma_{Z}$ can be understood by restricting attention to $Z_{2}-\Sigma_{Z_{2}}$ and even further, by restricting attention to $Z_{2, j}$. More precisely, the obstructions to extending $\underline{\omega}_{Z}$ can be 
understood by restricting attention to the very special case of a cobordism of simple type (as in Definition 2.2).

With the preceding understood, agree, for the remainder of Section 11, to restrict attention to a particular cobordism of simple type. Simplify notation by using $W$ now to denote this simple type cobordism. Then, $Z \subset W \times W$ and $\Sigma_{Z} \subset Z$ are defined accordingly:

With $Z$ as just redefined, note that the extension obstruction may well exist because $\operatorname{rank}\left(H^{2}\left(\Sigma_{Z}\right)\right) \geq 10$ while $\operatorname{rank}\left(H^{2}(Z)\right)=4$. Indeed, Lemma 2.3 describes $H^{2}(Z)\left(\approx \mathbb{R}^{4}\right)$, while Meyer-Vietoris with Proposition 5.2 find

(6.1) $H^{2}\left(\Sigma_{Z}\right) \approx H \equiv H^{2}\left(\Delta_{Z}\right) \oplus H^{2}\left(E_{L}\right) \oplus H^{2}\left(E_{R}\right) \oplus H^{2}\left(E_{-}\right) \oplus H^{2}\left(E_{+}\right)$.

In fact, the restriction map from $Z$ to $\Sigma_{Z}$ maps $H^{2}(Z)$ injectively into $H^{2}\left(E_{L}\right) \oplus$ $H^{2}\left(E_{R}\right)$.

c) Analyzing the obstruction.

Let $W$ be a cobordism of simple type and let $Z \subset W \times W$ and let $\Sigma_{Z} \subset Z$ be defined accordingly. Let $N_{Z} \subset Z$ be a regular neighborhood of $\Sigma_{Z}$. Introduce

$$
\begin{aligned}
& i_{1}^{*}: H^{2}\left(Z-\Sigma_{Z}\right) \longrightarrow H^{2}\left(N_{Z}-\Sigma_{Z}\right) \text { and } \\
& i_{2}^{*}: H^{2}\left(N_{Z}\right) \longrightarrow H^{2}\left(N_{Z}-\Sigma_{Z}\right)
\end{aligned}
$$

to denote the pull-back homomorphisms. One can conclude from the MeyerVietoris exact sequence that

$$
\underline{\omega}_{Z}=i_{1}^{*} \alpha+i_{2}^{*} \beta
$$

and the purpose of the subsequent arguments is to prove

Proposition 6.1. Equation (6.3) can be solved with a closed 2-form $\beta$ on $N_{Z}$ which obeys $\beta \wedge \beta=0$ and which vanishes near $\partial Z$.

Remark that the lemma implies that $\omega_{Z} \equiv \underline{\omega}_{Z}-i_{2}^{*} \beta$ extends over $Z-\Sigma_{Z}$ (as $\alpha$ in (6.3)) and it obeys $\omega_{Z} \wedge \omega_{Z} \wedge \omega_{Z}=0$ near $\Sigma_{Z}$ as required.

\section{d) Strategy for the proof of Proposition 6.1.}

The proof of Proposition 6.1 starts with the remark that the various framings that were introduced in the construction of $\omega_{Z}$ can be used to construct a homomorphism

$$
j: H_{2}\left(N_{Z}\right) \longrightarrow H_{2}\left(N_{Z}-\Sigma_{Z}\right)
$$

with the property that the composition of $j$ with $i_{2}$ (the dual of $i_{2}^{*}$ in (6.2)) gives the identity. Indeed, each of $\Delta_{Z}, E_{L, R}$ and $E 4 \pm$ have natural trivializations of their normal bundles. And, these trivializations can be used to push-off the generating cycles for the homology groups in question. (For $E_{L, R}$, see the proof of Lemma 4.4, and see Assertion 9 of Proposition 5.2 for $E_{4 \pm}$.) In this regard, note that an application of Meyer-Vietoris shows that the dimension 2 homology 
of $\Sigma_{Z}$ can be represented by submanifolds in $\Delta_{Z}, E_{L, R}$ and in the smooth parts of $E_{ \pm}$; and these submanifolds can be assumed to be disjoint from $E_{ \pm} \cap \Delta_{Z}$ and from $E_{L, R} \cap \Delta_{Z}$.

The homomorphism $\mathrm{j}$ has the property that

$$
\left\langle\omega_{Z}, j(\cdot)\right\rangle=0
$$

(This is because $\mathrm{j}$ is defined by the same homotopy class of normal framing which is used to define $\omega_{Z}$.)

Put (6.5) aside for the moment to consider the composition

$$
H_{2}\left(\Sigma_{Z}\right) \stackrel{j}{\longrightarrow} H_{2}\left(N_{Z}-\Sigma_{Z}\right) \stackrel{i_{1}}{\longrightarrow} H_{2}\left(Z-\Sigma_{Z}\right),
$$

which will be denoted by $\iota^{\prime}$. (The arrow $i_{1}$ in (6.6) is induced by the inclusion.) Define $Q \subset H^{2}\left(\Sigma_{Z}\right)$ by the exact sequence

$$
0 \longrightarrow Q \longrightarrow H^{2}\left(\Sigma_{Z}\right) \longrightarrow \operatorname{ker}\left(\iota^{\prime}\right)^{*} \longrightarrow 0
$$

Note that the restriction induced monomorphism $H^{2}(Z) \longrightarrow H^{2}\left(\Sigma_{Z}\right)$ factors through $Q$.

If the quotient $Q / H^{2}(Z)$ is zero, then it follows from (6.7) that (6.3) can be solved with $\beta \equiv 0$. If the quotient $Q / H^{2}(Z)$ is one dimensional, and if a generator can be represented by a form $\beta$ with $\beta \wedge \beta=0$, then Proposition 6.1 follows.

Thus, the proof of Proposition 6.1 will proceed with a proof that the dimension of $Q / H^{2}(Z)$ is one or less. The proof will end by finding a generator (when $\operatorname{dim}\left(Q / H^{2}(Z)\right)=1$ ) which is represented in $Q$ by a form with square zero (see (6.10), below).

By the way, the following lemma will be the principle tool for finding closed forms with square zero:

Lemma 6.2. Let $X$ be an oriented 4-manifold, and let $R \subset X$ be a compact, oriented, embedded surface. Suppose that $R$ has zero self-intersection number. Given an open neighborhood $O \subset X$ of $R$, there is a closed 2-form $\mu$ with $\mu \wedge \mu=0$ which is supported in $O$ and which represents the Poincaré dual to $R$ in $H_{\text {comp }}^{2}(X)$.

Proof. The surface $R$ has trivial normal bundle. Use this fact as in (6.12) of [7] to define a fibration from a neighborhood of $R$ in $X$ to the unit disk in $\mathbb{R}^{2}$ which sends $R$ to the origin. Use such a map to pull-back from said unit disk a 2 -form with compact support in the interior and with total mass equal to one. Set $\mu$ equal to this pull-back.

e) The dimension of $Q / H^{2}(Z)$.

Here is the answer to the dimension question:

LemMa 6.3. The dimension of $\left.Q / H^{2}(Z)\right)$ is one or zero.

Proof. The inclusion of $\Sigma_{Z}$ into $Z$ induces the homomorphism $\iota: H_{2}\left(\Sigma_{Z}\right) \longrightarrow$ $H_{2}(Z)$. Then, the dimension of $Q / H^{2}(Z)$ is equal to the dimension of $\operatorname{ker}(\iota) / \operatorname{ker}\left(\iota^{\prime}\right)$. 
To prove that $\operatorname{ker}(\iota) / \operatorname{ker}\left(\iota^{\prime}\right)$ has dimension 1 or less, consider an integral class $\sigma \in H_{2}\left(\Sigma_{Z}\right)$ with $\iota \cdot \sigma=0$, but with $\iota^{\prime} \cdot \sigma \neq 0$. Since $\iota \cdot \sigma=0$, there is a bounding 3 -cycle $\tau \subset Z$. The cycle $\tau$ is a sum of singular simplices; and these simplices can be chosen to have the following property: Each is a smooth map from the standard 3-simplex into $Z$ which is transversal to each of $\Delta_{Z}, E_{L}, E_{R}, E_{4 \pm}$ on the interior of every codimension $p=0, \cdots, 3$ face of the standard simplex. (Thus, the boundary of the standard simplex is mapped into the compliment of $\Sigma_{Z}$.) With this understood, it makes sense to speak of the intersection number of $\tau$ with each of $\Delta_{Z}, E_{L}, E_{R}, E_{4 \pm}$. Note that the intersection number between $\tau$ and $E_{4 \pm}$ can be assumed to be divisible by the integer $N$ of Proposition 5.2. This can be achieved by replacing $\sigma$ with $N \cdot \sigma$.

Observe now that intersections of $\tau$ with any of $E_{L}, E_{R}, E_{4 \pm}$ can be removed by changing $\tau$ to $\tau^{\prime}$, where $\tau^{\prime}$ has extra intersections with $\Delta_{Z}$. For example, one can add to $\tau$ some multiple of $\left[p_{0} \times M_{0}\right]$ to remove the intersection points with $E_{L}$ at the expense of adding such points to $\Delta_{Z}$. Likewise, adding to $\tau$ multiples of $\left[S^{3}\right]_{b_{1}}$ - will remove intersections with $E_{4-}$ and add intersections with $\Delta_{Z}$. Note that all of $\tau$ 's intersections with $E_{4-}$ can be transferred to $\Delta_{Z}$ because $E_{4-}$ is connected, and because $\tau$ 's intersection number with $\Delta_{Z}$ is assumed divisible by the integer $N$ from Proposition 5.2. The cases for $E_{R}$ and $E_{4 \pm}$ are analogous. (See, e.g., $(9.9 \mathrm{a}, \mathrm{b})$ in [7].)

It follows from the preceding that $\operatorname{ker}(\iota) / \operatorname{ker}\left(\iota^{\prime}\right)$ is at most one-dimensional. This is because any element in this quotient can be represented by a closed 2-cycle which bounds $\tau$ as above, whose intersections with $\Sigma_{Z}$ lie in $\Delta_{Z}$ only. Given two such elements, a non-trivial linear combination would be represented by a closed 2-cycle which bounds $\tau$ as above with absolutely no intersections with $\Sigma_{Z}$. Such a linear combination would be zero in $\operatorname{ker}(\iota) / \operatorname{ker}\left(\iota^{\prime}\right)$.

\section{f) $\operatorname{ker}(\iota)$.}

The final step in the proof of Proposition 6.1 is to consider the possible generators of $Q / H^{2}(Z)$ in the case where this group has dimension 1 .

A generator of this group is represented by a class $\ell \in H^{2}\left(\Sigma_{Z}\right)$ which annihilates the kernel of $\iota^{\prime}$, but which is non-zero on a class $\sigma \in H_{2}\left(\Sigma_{Z}\right)$ which is annihilated by $\iota$ but not by $\iota^{\prime}$. The analysis of $\ell$ proceeds by considering various possibilities for $\operatorname{ker}(\iota) / \operatorname{ker}\left(\iota^{\prime}\right)$. Remark that if this group has dimension 1 , then it can be represented in $\operatorname{ker}(\iota)$ by some generator.

In $H_{2}\left(\Delta_{Z}\right) \oplus H_{2}\left(E_{L}\right) \oplus H_{2}\left(E_{R}\right)$ sits a two-dimensional subspace of $\operatorname{ker}(\iota)$. An element in $\operatorname{ker}(\iota) \cap\left(H_{2}\left(\Delta_{Z}\right) \oplus H_{2}\left(E_{L}\right) \oplus H_{2}\left(E_{R}\right)\right)$ has the form

$$
\sigma=\sigma_{\Delta}-\sigma_{L}-\sigma_{R}
$$

where $\sigma$. sits in the summand with the corresponding label. Here, each $\sigma$. pushes forward to $W$ as the same class $\sigma_{0} \in H_{2}(W)$. Then, two generators of the kernel of $\iota$ in $H_{2}\left(\Delta_{Z}\right) \oplus H_{2}\left(E_{L}\right) \oplus H_{2}\left(E_{R}\right)$ are given by $\sigma$ as above with $\sigma_{0}=\left[T_{1}\right]$ and with $\sigma_{0}=\left[T_{2}\right]$. 
The remaining generators of the kernel of $\iota$ can be taken to have the form

$$
\begin{array}{ll}
\text { 1) } & \sigma_{+1}-\sigma_{R 1} \text { and } \sigma_{+2}-\sigma_{L 2} \\
\text { 2) } & \sigma_{-1}-\sigma_{L 1} \text { and } \sigma_{-2}-\sigma_{R 2} \\
\text { 3) } & \left\{\lambda_{ \pm c}\right\} .
\end{array}
$$

Here, $\sigma_{ \pm 1,2} \in H_{2}\left(E_{ \pm}\right)$, while $\sigma_{L 1,2} \in H_{2}\left(E_{L}\right)$ and $\sigma_{R 1,2} \in H_{2}\left(E_{R}\right)$ project to $H_{2}(W)$ as multiples of $\left[T_{1,2}\right]$, respectively. Meanwhile, $\left\{\lambda_{ \pm c}\right\} \in H_{2}\left(E_{ \pm}\right)$is a finite set of classes, and each is represented by a push-off of some $T_{\alpha, \beta \pm}$ as described in (4.23).

Lemma 6.4. The classes in (6.9) are annihilated by $\iota^{\prime}$.

Proof. This follows from Assertion 9 of Proposition 5.2.

With the preceding lemma understood, it follows that a generator of $\operatorname{ker}(\iota) / \operatorname{ker}\left(\iota^{\prime}\right)$ is described by $(6.8)$.

\section{g) If $\sigma=\sigma_{\Delta}-\sigma_{L}-\sigma_{R}$ is not annihilated by $\iota^{\prime}$.}

In this case, there exists $\sigma$ as above with either $\sigma_{0}=\left[T_{1}\right]$ or $\sigma_{0}=\left[T_{2}\right]$. For arguments sake, assume $\sigma_{0}=\left[T_{1}\right]$. Let $\beta_{\Delta_{1}} \in H^{2}\left(E_{\Delta}\right)$ be the pull-back by the map $\pi_{L}$ to $W$ of the Poincaré dual to $\left[T_{2}\right]$. Then $\beta_{\Delta_{1}}$ pairs non-trivially with $\sigma_{L}$ and so with $\sigma$. Let $\beta_{R 2} \in H^{2}\left(E_{R}\right)$ be the pull-back by $\pi_{R}$ of the Poincaré dual to $\left[T_{1}\right]$. Note that $\beta_{R 2}$ pairs trivially with $\sigma$.

It follows that there is $c \in \mathbb{R}$ such that $\beta^{\prime} \equiv \beta_{\Delta_{1}}+c \cdot \beta_{R 2}$ annihilates the $\operatorname{ker}(\iota)$ in $H_{2}\left(\Delta_{Z}\right) \oplus H_{2}\left(E_{L}\right) \oplus H_{2}\left(E_{R}\right)$. This $\beta^{\prime}$ will have trivial pairing with the classes in (6.9.1), and it will have trivial pairing with $\sigma_{-1}-\sigma_{L 1}$ in (6.9.2), but unless $c=0$, it will pair non-trivially with $\sigma_{-2}-\sigma_{R 2}$ in (6.9.2). However, note that the Poincaré dual, $\beta_{-2} \in H_{2}\left(E_{-}\right)$, to $\sigma_{-1}$ pairs trivially with $\sigma_{-1}$ and non-trivially with $\sigma_{-2}$. And so, there is a real number $c^{\prime}$ such that

$$
\beta \equiv \beta_{\Delta_{1}}+c \cdot \beta_{R 2}+c^{\prime} \cdot \beta_{-2}
$$

annihilates all of the classes in (6.9.2). Note that $\beta$ will also annihilate the classes in (6.9.3).

By appeal to Lemma 6.2 , each of $\beta_{\Delta_{1}}, \beta_{R 2}$, and $\beta_{-2}$ can be represented by a closed form with square zero and with support away from $\partial Z$. (This is because $\left[T_{1}\right]$ and $\left[T_{2}\right]$ are classes with square zero in $W$.) Furthermore, Lemma 6.2 insures that these forms can be constructed to have disjoint supports. Thus, $\beta$ will vanish near $\partial Z$ and have square zero as required.

\section{REFERENCES}

[1] S. Axelrod \& I. M. Singer, Chern-Simons perturbation theory, Proc. XXth DGM Conference (New York, 1991, S. Catto and A. Tocha, eds.), World Scientific, 1992, 3-45.

[2] _ Chern-Simones perturbation theory. II, J. Differential Geometry 39 (1994) 173-213. 
[3] F. Hirzebruch, W. D. Neumann \& S. S. Koh, Differentiable manifolds and quadratic forms, Marcell Decker, New York, 1971.

[4] M. Kontsevich, Feynman diagrams and low dimensional topology, MaxPlanck-Institute, Bonn, Preprint, 1993.

[5] C. C. MacDuffe, The theory of matrices, Springer, Berlin, 1933.

[6] J. Milnor, Lectures on the h-cobordism theorem, Notes by L. Siebenmann and J. Sondau, Princeton University Press, Princeton, 1965.

[7] C. H. Taubes, Homology cobordisms and the simplest perturbative ChernSimons 3-manifold invariant, Geometry, Topology and Physics for Raovl Bott, ed. S.T. Yau, International Press, 1995.

[8] E. Witten, Quantum field theory and the Jones polynomial, Comm. Math. Phys. 117 (1988) 351-399. 